CRYSTALLOGRAPHIC COMMUNICATIONS

ISSN 2056-9890

Received 9 March 2017

Accepted 17 March 2017

Edited by C. Rizzoli, Universita degli Studi di Parma, Italy

Keywords: crystal structure; platinum(II) complexes; 2-aminopyridine; eugenol.

CCDC references: $1538567 ; 1538566$

Supporting information: this article has supporting information at journals.iucr.org/e

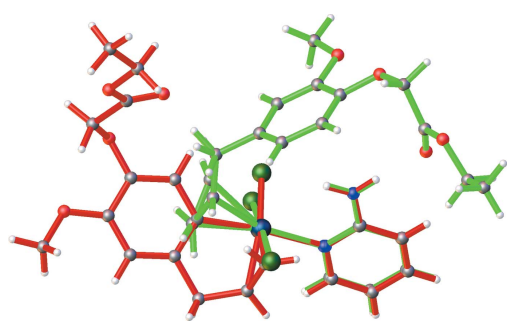

OPEN $\odot$ ACCESS

\section{Crystal structures of two platinum(II) complexes containing ethyl eugenoxyacetate and 2-amino- pyridine}

\author{
Hai Le Thi Hong, ${ }^{a}$ Thao Nguyen Thu, ${ }^{a}$ Hien Nguyen ${ }^{a}$ and Luc Van Meervelt ${ }^{\text {* } *}$ \\ ${ }^{\mathbf{a}}$ Department of Chemistry, Hanoi National University of Education, 136 Xuan Thuy, Cau Giay, Hanoi, Vietnam, and \\ ${ }^{\mathbf{b}}$ Department of Chemistry, KU Leuven, Biomolecular Architecture, Celestijnenlaan 200F, Leuven (Heverlee), B-3001, \\ Belgium. *Correspondence e-mail: luc.vanmeervelt@kuleuven.be
}

In the title complexes, trans-(2-aminopyridine- $\kappa N)$ dichlorido\{4-ethoxycarbonylmethoxy-3-methoxy-1-[(2,3- $\eta$ )-prop-2-en-1-yl]benzene)platinum(II), $\quad\left[\mathrm{PtCl}_{2}-\right.$ $\left.\left(\mathrm{C}_{5} \mathrm{H}_{6} \mathrm{~N}_{2}\right)\left(\mathrm{C}_{14} \mathrm{H}_{18} \mathrm{O}_{4}\right)\right]$, (I), and (2-aminopyridine- $\left.\kappa N\right)$ chlorido $\{5$-ethoxycarbonylmethoxy-4-methoxy-1-[(2,3- $\eta$ )-prop-2-en-1-yl]phenyl- $\left.\kappa C^{1}\right\}$ platinum(II), [Pt$\left.\left(\mathrm{C}_{14} \mathrm{H}_{17} \mathrm{O}_{4}\right) \mathrm{Cl}\left(\mathrm{C}_{5} \mathrm{H}_{6} \mathrm{~N}_{2}\right)\right]$, (II), the central $\mathrm{Pt}^{\mathrm{II}}$ metal atom displays a distorted square-planar coordination, with the $\mathrm{Pt}^{\mathrm{II}}$ atom coordinated by the pyridine $\mathrm{N}$ atom, the $\mathrm{C}=\mathrm{C}$ double bond of the eugenol ligand and two $\mathrm{Cl}$ atoms for (I) or one $\mathrm{Cl}$ atom and a $\mathrm{C}$ atom of the phenyl ring for (II). The allyl fragment in (I) is disordered, with population parameters 0.614 (14) and 0.386 (14) for the two positions of the central $\mathrm{C}$ atom. The least-squares planes through the two aromatic ring systems make a dihedral angle of $51.10(13)^{\circ}$ for (I) and $78.5(2)^{\circ}$ for (II). Intramolecular $\mathrm{N}-\mathrm{H} \cdots \mathrm{O}$ and $\mathrm{N}-\mathrm{H} \cdots \pi$ interactions occur in (I). In (I), inversion dimers formed by $\mathrm{C}-\mathrm{H} \cdots \mathrm{Cl}$ interactions are further linked into chains parallel to the $b$ axis by $\mathrm{C}-\mathrm{H} \cdots \mathrm{O}$ hydrogen bonds. Both aromatic rings are involved in $\pi-\pi$ interactions, with centroid-to-centroid distances of 3.508 (3) and 3.791 (3) $\AA$. In (II), inversion dimers form chains parallel to the $b$ axis by $\mathrm{C}-\mathrm{H} \cdots \mathrm{O}$ interactions.

\section{Chemical context}

Since the discovery of the anticancer activity and subsequent clinical success of cisplatin $\left\{\right.$ cis- $\left.\left[\mathrm{PtCl}_{2}\left(\mathrm{NH}_{3}\right)_{2}\right]\right\}$, platinumbased compounds have been widely synthesized and studied as potential chemotherapeutic agents (Wong \& Giandomenico, 1999). Despite the great success in treating certain kinds of cancer, there are several side effects, and both intrinsic and acquired resistance limit the organotropic profile of the drug (Chabner \& Roberts, 2005; Kelland, 2007; Wilson \& Lippard, 2014). Hence, there is continuing interest in the development of new platinum complexes that have high activities but low toxicity (Johnstone et al., 2014).

Several natural arylolefins, such as safrole (in sassafras oil), eugenol (in clove oil) and anethole (in anise and fennel oil), and their derivatives have been used as important intermediate materials to synthesize many compounds that have various applications in the flavouring, food and pharmaceutical industries (Jadhav et al., 2004). Recently, a number of $\mathrm{Pt}^{\mathrm{II}}$ complexes containing natural arylolefins as ligands, i.e. safrole or derivatives of eugenol such as methyleugenol and alkyleugenoxyacetate, have been prepared (Da et al., 2010, 2012; Da, Chi et al., 2015; Da, Hai et al., 2015; Nguyen Thi Thanh et al., 2016; Le Thi Hong et al., 2016). The insertion of these natural arylolefins into the coordination with $\mathrm{Pt}^{\mathrm{II}}$ and their transformations formed complexes with novel structures and 
high applicability. In particular, many of these organoplatinum(II) complexes exhibit significant inhibitory activities against human cancer cells (Da et al., 2012; Da, Chi et al., 2015; Da, Hai et al., 2015).

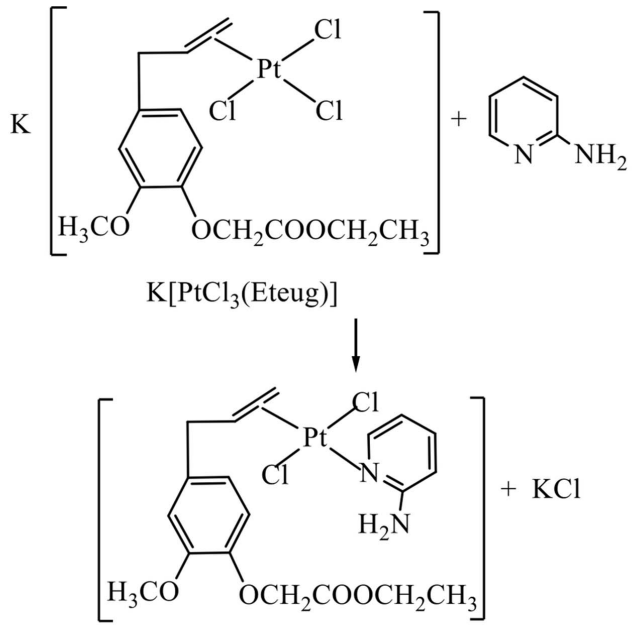

(I)<smiles>CCOC(=O)COc1cc2c(cc1OC)Cc1cc(OC)c(OC)cc1[P+]2(Cl)C[Te]1(Cl)C=CCc2cc(OC)c(OC)cc21</smiles>

$[\mathrm{PtCl}(\text { Eteug- } 1 \mathrm{H})]_{2}$<smiles>CCOC(=O)OCc1cc(OC)c(OCC)cc1CP(Cl)[n+]1ccccc1N</smiles>

(II)

Herein, we report the syntheses and crystal structure determinations of organoplatinum(II) complexes formed by the complexation of 2-aminopyridine as ligand with the mononuclear platinum(II) complex $\mathrm{K}\left[\mathrm{PtCl}_{3}(\right.$ Eteug) $]$ and the binuclear platinum(II) complex $\left[\mathrm{Pt}_{2}(\text { Eteug- } 1 \mathrm{H})_{2} \mathrm{Cl}_{2}\right]$ (Eteug is ethyleugenoxylacetate).

\section{Structural commentary}

In both title complexes, the central $\mathrm{Pt}^{\mathrm{II}}$ metal atom displays a distorted square-planar coordination (Fig. 1). In addition to the two $\mathrm{Cl}$ atoms in dichloride complex (I), the pyridine $\mathrm{N}$ atom and the $\mathrm{C}=\mathrm{C}$ double bond of the eugenol ligand coordinate to the central $\mathrm{Pt}^{\mathrm{II}}$ atom. In monochloride complex (II),

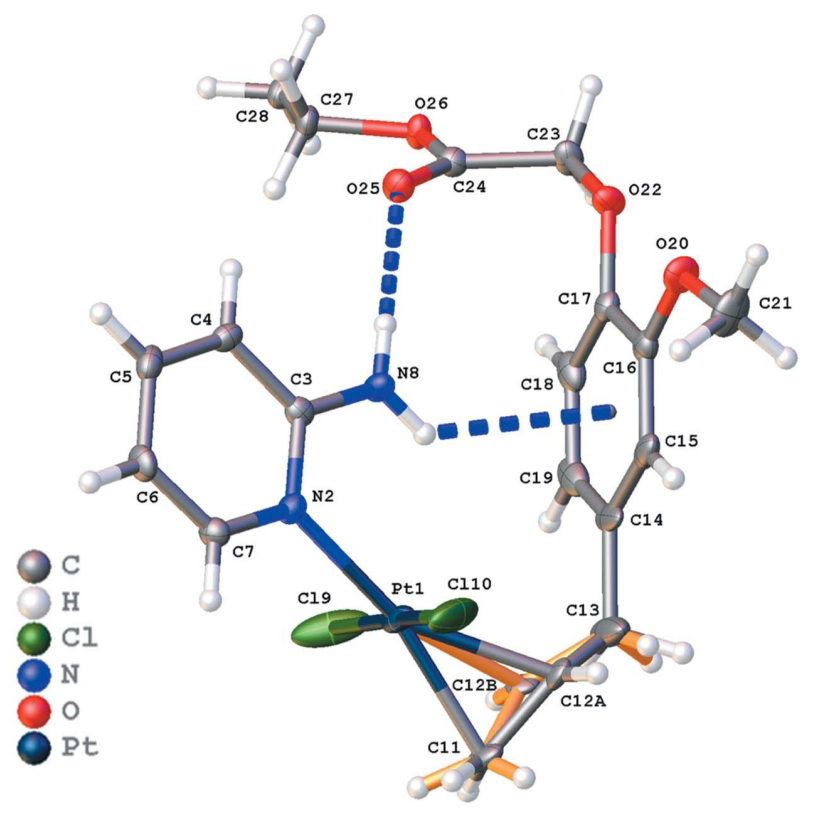

(a)

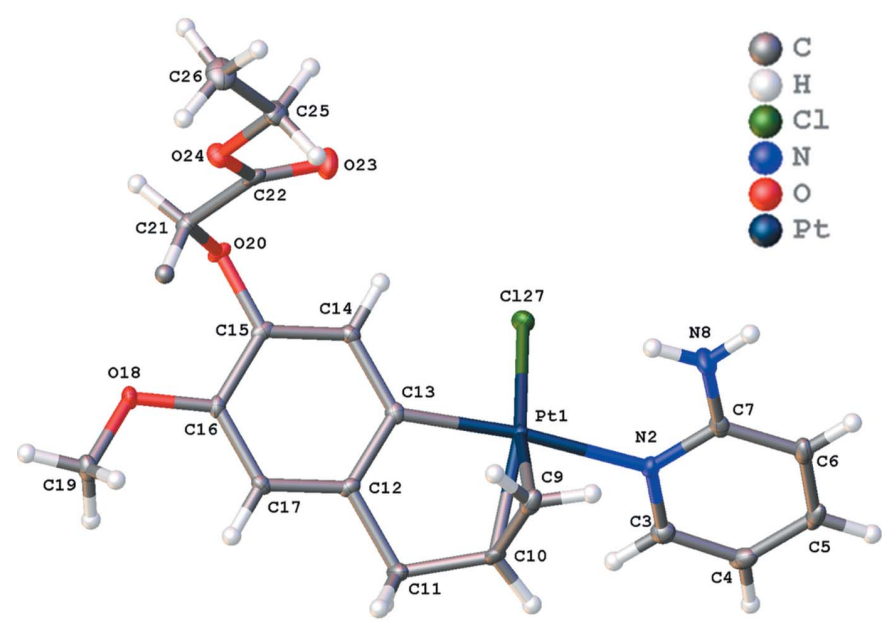

(b)

Figure 1

Views of the asymmetric units in $(a)$ (I) and (b) (II), showing the atomlabelling schemes. Displacement ellipsoids are drawn at the $50 \%$ probability level. Orange bonds in $(a)$ show the allyl fragment with a population parameter of $0.386(14)$. Blue dotted lines indicate intramolecular interactions in $(a)$.

one $\mathrm{Cl}$ atom is replaced by a $\mathrm{C}$ atom of the eugenol phenyl group. An overlay of the $\mathrm{Pt}$-2-amino fragment present in both structures clearly shows the differences in coordination (Fig. 2). Where in (I) the $\mathrm{Cl}$ atoms are trans with respect to each other, this is the case for the two aromatic rings in (II). One $\mathrm{Cl}$ and the $\mathrm{C}=\mathrm{C}$ coordinations in (I) are replaced by, respectively, $\mathrm{C}=\mathrm{C}$ and a phenyl $\mathrm{C}$ atom in (II). In both cases, the 2-aminopyridine ligand only interacts via the ring $\mathrm{N}$ atom. In (I), the $\mathrm{CH}_{2}-\mathrm{CH}=\mathrm{CH}_{2}$ fragment is disordered, with population parameters of 0.614 (14) and 0.386 (14) for the two positions of the central $\mathrm{C}$ atom. The dihedral angles between the planes through the two aromatic rings are 78.5 (2) and 


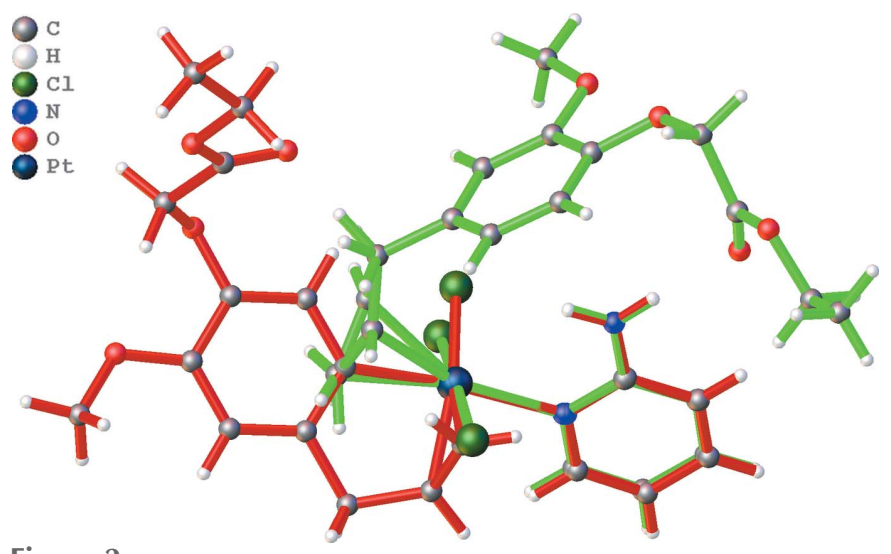

Figure 2

Overlay of the Pt-2-aminopyridine fragment present in (I) (green bonds) and (II) (red bonds).

$51.10(13)^{\circ}$ for (I) and (II), respectively. In (I), the $\mathrm{H}$ atoms of the amino group are involved in a weak intramolecular $\mathrm{N}-$ $\mathrm{H} \cdots \mathrm{O}$ interaction $(\mathrm{N} 8-\mathrm{H} 8 B \cdots \mathrm{O} 25$, Table 1$)$ and an $\mathrm{N}-$ $\mathrm{H} \cdots \pi$ interaction $(\mathrm{N} 8-\mathrm{H} 8 A \cdots C g 1$, Table $1 ; C g 1$ is the centroid of the C14-C19 ring). Similar interactions are not possible in (II) due to the different orientation of the ligands.

\section{Supramolecular features}

The complexes crystallize in different space groups, viz. $P \overline{1}$ for dichloride complex (I) and $P 2_{1} / c$ for monochloride complex (II).

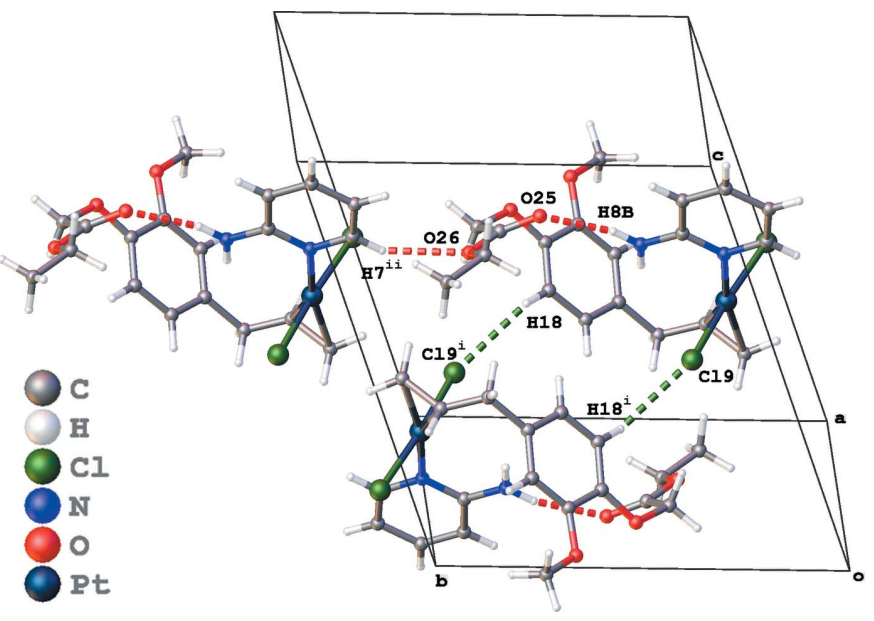

Figure 3

Packing diagram of (I), showing the $\mathrm{C}-\mathrm{H} \cdots \mathrm{O}$ (red dotted lines) and $\mathrm{C}-$ $\mathrm{H} \cdots \mathrm{Cl}$ interactions (green dotted lines). [Symmetry codes: (i) $-x,-y+1$, $-z+1$; (ii) $x, y-1, z$.]

The crystal packing of (I) is dominated by hydrogen bonding and $\pi-\pi$ interactions. Inversion dimers formed by $\mathrm{C} 18-\mathrm{H} 18 \cdots \mathrm{Cl} 9^{\mathrm{i}}$ hydrogen bonds are further linked into chains parallel to the $b$ axis by $\mathrm{C} 7-\mathrm{H} 7 \cdots \mathrm{O} 26^{\mathrm{ii}}$ hydrogen bonds [Table 1 and Fig. 3; symmetry codes: (i) $x, y-1$, $z$; (ii) $-x,-y+1,-z+1]$. Both aromatic rings show $\pi-\pi$ stacking, with $C g 1 \cdots C g 1^{\text {iii }}=3.791$ (3) $\AA$ for the phenyl ring and $C g 2 \cdots C g 2^{\text {iv }}=3.508(3) \AA$ for the pyridine ring $[C g 1$ and $C g 2$ are the centroids of the $\mathrm{C} 14-\mathrm{C} 19$ and N2/C3-C7 rings;

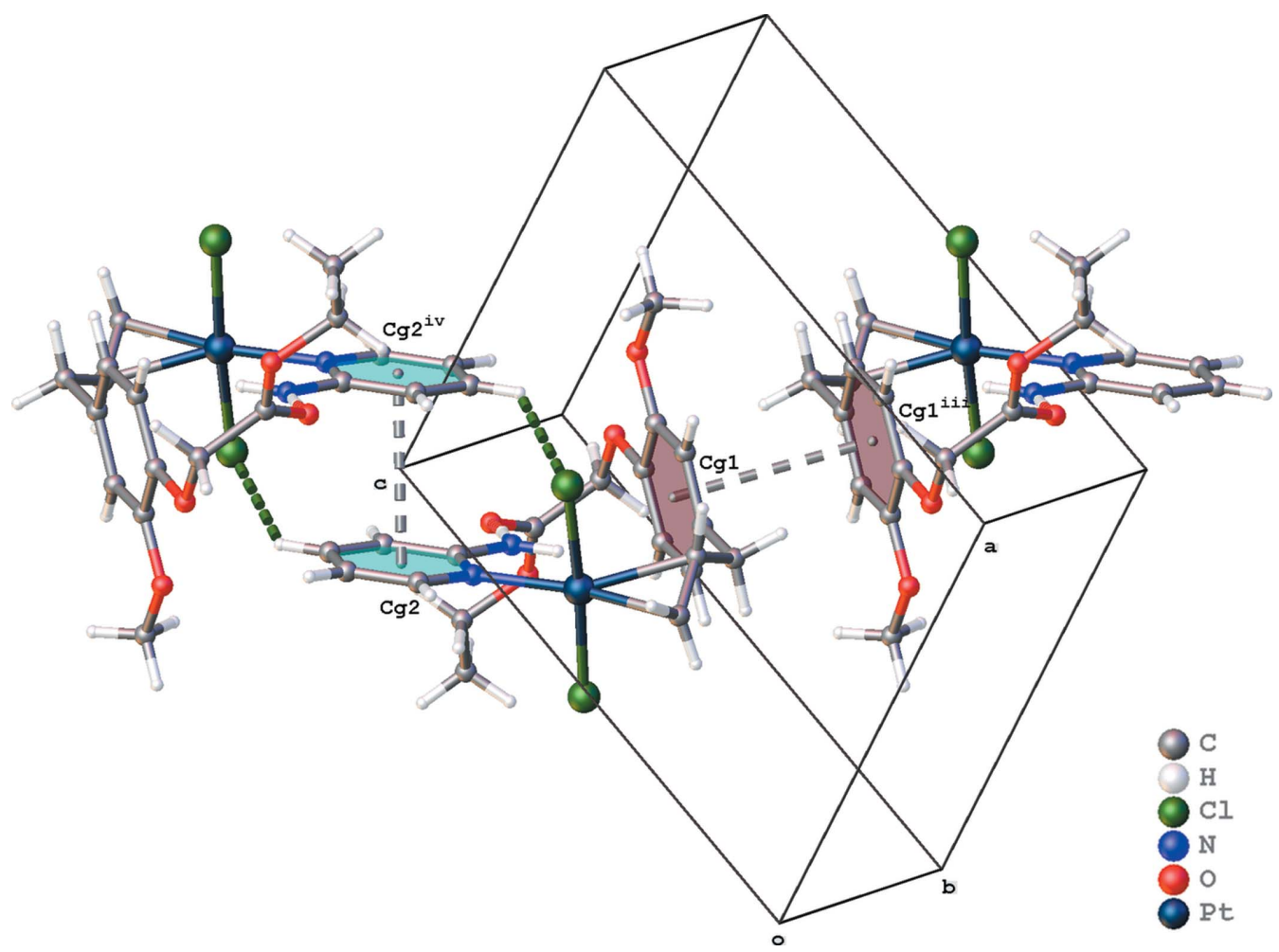

Figure 4

Partial packing diagram of (I), showing $\pi-\pi$ stacking (gray dotted lines). [ $C g 1$ and $C g 2$ are the centroids of the C14-C19 and N2/C3-C7 rings; symmetry codes: (iii) $-x+1,-y+1,-z+1$; (iv) $-x,-y,-z+2$.] 
Table 1

Hydrogen-bond geometry $\left(\AA,^{\circ}\right)$ for (I).

$C g 1$ is the centroid of the $\mathrm{C} 14-\mathrm{C} 19$ ring.

\begin{tabular}{lllll}
\hline$D-\mathrm{H} \cdots A$ & $D-\mathrm{H}$ & $\mathrm{H} \cdots A$ & $D \cdots A$ & $D-\mathrm{H} \cdots A$ \\
\hline $\mathrm{N} 8-\mathrm{H} 8 B \cdots \mathrm{O} 25$ & 0.88 & 2.19 & $3.054(5)$ & 167 \\
$\mathrm{C} 7-\mathrm{H} 7 \cdots \mathrm{O} 26^{\mathrm{i}}$ & 0.95 & 2.49 & $3.258(6)$ & 138 \\
$\mathrm{C} 18-\mathrm{H} 18 \cdots \mathrm{C} 9^{\mathrm{ii}}$ & 0.95 & 2.78 & $3.460(5)$ & 130 \\
$\mathrm{~N} 8-\mathrm{H} 8 A \cdots C g 1$ & 0.88 & 2.53 & $3.166(4)$ & 129 \\
\hline
\end{tabular}

Symmetry codes: (i) $x, y-1, z$; (ii) $-x,-y+1,-z+1$.

symmetry codes: (iii) $-x+1,-y+1,-z+1$; (iv) $-x,-y$, $-z+2$; Fig. 4].

The crystal packing of (II) is built up by $\mathrm{C}-\mathrm{H} \cdots \mathrm{O}, \mathrm{N}-$ $\mathrm{H} \cdots \mathrm{Cl}$ and $\mathrm{C}-\mathrm{H} \cdots \pi$ interactions (Table 2 and Fig. 5). Two types of inversion dimers are created by $\mathrm{C}-\mathrm{H} \cdots \mathrm{O}$ interactions enclosing $R_{2}^{2}(10)$ and $R_{2}^{2}(16)$ ring motifs, and resulting in the formation of chains parallel to the $b$ axis. No $\pi-\pi$ interactions are observed in the packing of (II).

\section{Database survey}

The Pt $-\mathrm{N}$ distances of 2.066 (3) $\AA$ in (I) and 2.143 (2) $\AA$ in (II) agree well with the average $\mathrm{Pt}-\mathrm{N}$ distance of 2.06 (7) $\AA$ for $\mathrm{Pt}$-pyridine fragments present in the Cambridge Structural Database (CSD, Version 5.38, last update February 2017; Groom et al., 2016).

The CSD contains $34 \mathrm{Pt}$ complexes with $\mathrm{Pt}$ coordinated by $\mathrm{Cl}$, pyridine and $\mathrm{C}=\mathrm{C}$, with 28 complexes having an additional $\mathrm{Cl}$ atom as the fourth ligand (27 trans and one cis coordination), three a $\mathrm{C}$ atom and another three an $\mathrm{N}$ atom.
Table 2

Hydrogen-bond geometry $\left(\AA,^{\circ}\right)$ for (II).

$\mathrm{Cg} 2$ is the centroid of the $\mathrm{C} 12-\mathrm{C} 17$ ring.

\begin{tabular}{lllll}
\hline$D-\mathrm{H} \cdots A$ & $D-\mathrm{H}$ & $\mathrm{H} \cdots A$ & $D \cdots A$ & $D-\mathrm{H} \cdots A$ \\
\hline $\mathrm{C} 17-\mathrm{H} 17 \cdots \mathrm{O} 24^{\mathrm{i}}$ & 0.95 & 2.60 & $3.501(3)$ & 159 \\
$\mathrm{C} 25-\mathrm{H} 25 B \cdots \mathrm{O} 23^{\mathrm{ii}}$ & 0.99 & 2.60 & $3.449(3)$ & 144 \\
$\mathrm{~N} 8-\mathrm{H} 8 B \cdots \mathrm{Cl} 27^{\mathrm{iii}}$ & 0.88 & 2.67 & $3.413(2)$ & 143 \\
$\mathrm{C} 19-\mathrm{H} 19 A \cdots C g 2^{\mathrm{i}}$ & 0.98 & 2.63 & $3.476(3)$ & 145 \\
\hline
\end{tabular}

Symmetry codes: (i) $\quad-x+1,-y,-z+1$; (ii) $\quad-x+1,-y+1,-z+1$; $\quad$ (iii) $-x, y+\frac{1}{2},-z+\frac{1}{2}$.

The synthesis of (II), starting from the dinuclear complex $\left[\mathrm{Pt}_{2} \mathrm{Cl}_{2}(\text { Eteug-1H })_{2}\right]$, can be rationalized by the replacement of the $\mathrm{Cl}$ atom in the trans position with respect to the $\mathrm{C}=\mathrm{C}$ bond. Verification of the $\mathrm{Pt}-\mathrm{Cl}$ distances in the dinuclear complex di- $\mu$-chlorido-bis $\left[\left(\eta^{2}-2\right.\right.$-allyl-4-methoxy-5-\{[(propan2-yloxy)carbonyl $]$ methoxy\}phenyl- $\left.\kappa C^{1}\right)$ platinum(II)], $\quad\left[\mathrm{Pt}_{2}-\right.$ (IsoPreug- $1 \mathrm{H})_{2} \mathrm{Cl}_{2}$ ] (IsoPreug- $1 \mathrm{H}$ is isopropyleugenoxylacetate; CSD refcode EWAVIJ; Nguyen Thi Thanh et al., 2016) indicates that the longest $\mathrm{Pt}-\mathrm{Cl}$ bond $[2.4773$ (7) versus 2.3527 (7) $\AA$ ] is cleaved, leading to a cis position of 2 -aminopyridine with respect to the $\mathrm{C}=\mathrm{C}$ bond.

\section{Synthesis and crystallization}

\subsection{Synthesis of $\mathrm{K}\left[\mathrm{PtCl}_{3}(\right.$ Eteug) $]$ and $\left[\mathrm{Pt}_{2} \mathrm{Cl}_{2}(\text { Eteug-1 } \mathrm{H})_{2}\right]$}

The mononuclear complex $\mathrm{K}\left[\mathrm{PtCl}_{3}\right.$ (Eteug) $]$ and the dinuclear chelate ring complex $\left[\mathrm{Pt}_{2} \mathrm{Cl}_{2}(\text { Eteug- } 1 \mathrm{H})_{2}\right]$ were synthesized following the protocol of Da and co-workers (Da et al., 2012; Da, Chi et al., 2015; Da, Hai et al., 2015).

Figure 5

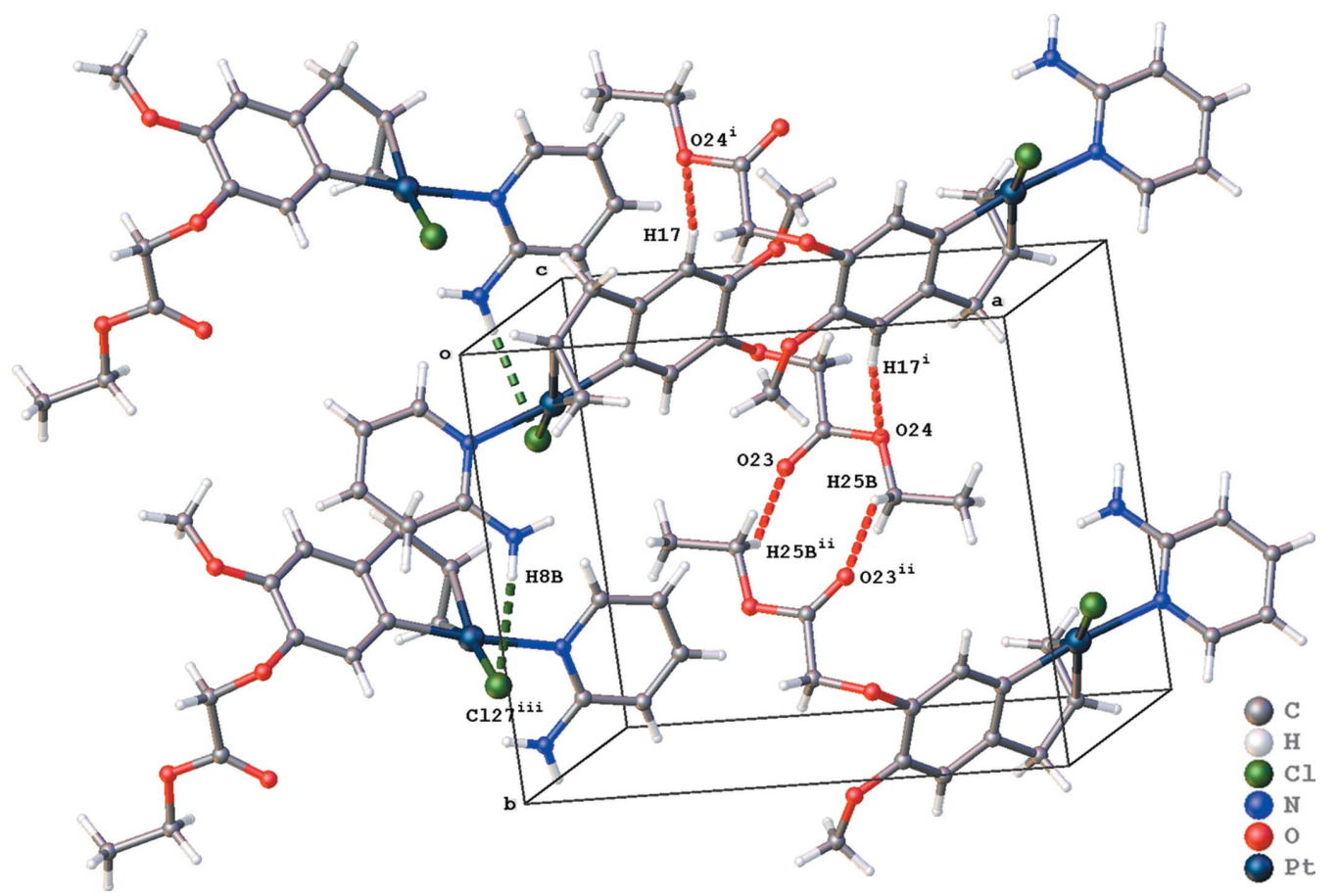

Partial packing diagram of (II), showing the $\mathrm{C}-\mathrm{H} \cdots \mathrm{O}$ (red dotted lines), $\mathrm{N}-\mathrm{H} \cdots \mathrm{Cl}$ (green dotted lines) and $\mathrm{C}-\mathrm{H} \cdots \pi$ (gray dotted lines) interactions. [Symmetry codes: (i) $-x+1,-y,-z+1$; (ii) $-x+1,-y+1,-z+1$; (iii) $-x, y+\frac{1}{2},-z+\frac{1}{2}$ ] 
Table 3

Experimental details.

(I)

Crystal data

Chemical formula

$M_{\mathrm{r}}$

Crystal system, space group

Temperature (K)

$a, b, c(\AA)$

$\alpha, \beta, \gamma\left({ }^{\circ}\right)$

$V\left(\AA^{3}\right)$

$Z$

Radiation type

$\mu\left(\mathrm{mm}^{-1}\right)$

Crystal size (mm)

Data collection

Diffractometer

Absorption correction

$T_{\min }, T_{\max }$

No. of measured, independent and observed

$[I>2 \sigma(I)]$ reflections

$R_{\text {int }}$

$(\sin \theta / \lambda)_{\max }\left(\AA^{-1}\right)$

Refinement

$R\left[F^{2}>2 \sigma\left(F^{2}\right)\right], w R\left(F^{2}\right), S$

No. of reflections

No. of parameters

No. of restraints

$\mathrm{H}$-atom treatment

$\Delta \rho_{\max }, \Delta \rho_{\min }\left(\mathrm{e} \AA^{-3}\right)$

\author{
$\left[\mathrm{PtCl}_{2}\left(\mathrm{C}_{5} \mathrm{H}_{6} \mathrm{~N}_{2}\right)\left(\mathrm{C}_{14} \mathrm{H}_{18} \mathrm{O}_{4}\right)\right]$ \\ 610.39 \\ Triclinic, $P \overline{1}$ \\ 100 \\ 8.3187 (3), 11.4119 (3), 12.1012 (4) \\ 70.437 (3), 73.688 (3), 87.038 (2) \\ $1037.76(6)$ \\ 2 \\ Mo $K \alpha$ \\ 7.05 \\ $0.30 \times 0.30 \times 0.15$
}

Agilent SuperNova diffractometer (single source at offset, Eos detector)

Multi-scan (CrysAlis PRO; Rigaku Oxford Diffraction, 2015)

$0.406,1.000$

$21329,4241,4006$

0.052

0.625

$0.029,0.065,1.04$

4241

265

26

$\mathrm{H}$-atom parameters constrained

$1.41,-2.25$
(II)

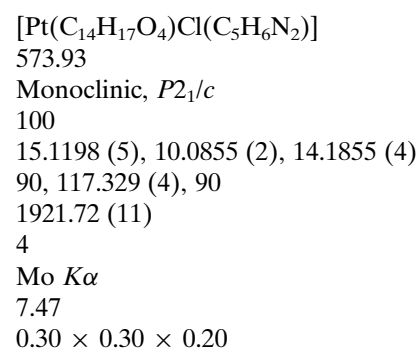

Agilent SuperNova diffractometer (single source at offset, Eos detector)

Multi-scan (CrysAlis PRO; Rigaku Oxford Diffraction, 2015)

$0.314,1.000$

20268, 3928, 3715

0.033

0.624

$0.016,0.038,1.06$

3928

246

0

$\mathrm{H}$-atom parameters constrained $0.69,-1.04$

Computer programs: CrysAlis PRO (Rigaku Oxford Diffraction, 2015), SHELXS97 (Sheldrick, 2008), SUPERFLIP (Palatinus \& Chapuis, 2007), SHELXL2014 (Sheldrick, 2015) and OLEX2 (Dolomanov et al., 2009).

\subsection{Synthesis of trans-[PtCl 2 (Eteug) $\left.\left(\mathrm{C}_{5} \mathrm{H}_{6} \mathrm{~N}_{2}\right)\right]$, (I)}

While stirring, a solution of 2-aminopyridine $(0.22 \mathrm{mmol})$ in acetone $(2 \mathrm{ml})$ was added slowly to a solution of $\mathrm{K}\left[\mathrm{PtCl}_{3^{-}}\right.$ (Eteug)] $(0.2 \mathrm{mmol})$ in acetone $(15 \mathrm{ml})$. After $2 \mathrm{~h}$, a white precipitate of $\mathrm{KCl}$ was separated out. After stirring for $3 \mathrm{~h}$ at room temperature, ethanol $(2 \mathrm{ml})$ was added to the obtained solution. Slow evaporation of the solvent at room temperature afforded the desired product as bright orange-yellow crystals. The yield was $80 \%$. The product is soluble in acetone and chloroform, but only slightly soluble in ethanol and insoluble in water. Single crystals suitable for X-ray diffraction were obtained from an acetone/ethanol $(3: 1 \mathrm{v} / \mathrm{v})$ solution via slow evaporation of the solvents at $277-278 \mathrm{~K}$.

\subsection{Data for trans- $\left[\mathrm{PtCl}_{2}\right.$ (Eteug) $\left.\left(\mathrm{C}_{5} \mathrm{H}_{6} \mathrm{~N}_{2}\right)\right]$, (I)}

IR (Impack-410 Nicolet spectrometer, $\mathrm{KBr}, \mathrm{cm}^{-1}$ ): 3454 , $3341\left(v_{\mathrm{NH}}\right) ; 3060,2930\left(v_{\mathrm{CH}}\right) ; 1739\left(v_{\mathrm{C}=\mathrm{O}}\right) ; 1598,1512$ (aromatic, $v_{\mathrm{C}=\mathrm{C}}, v_{\mathrm{C}=\mathrm{N}}$ ).

${ }^{1} \mathrm{H}$ NMR ( $\delta$ p.p.m.; Bruker AVANCE $500 \mathrm{MHz}, \mathrm{CDCl}_{3}$ ): $7.15\left(1 \mathrm{H}, d,{ }^{4} J=1.5 \mathrm{~Hz}, \mathrm{Ar}\right), 7.00\left(1 \mathrm{H}, t,{ }^{3} J=8.0 \mathrm{~Hz},{ }^{4} J=\right.$ $1.5 \mathrm{~Hz}, \mathrm{Ar}), 6.77\left(1 \mathrm{H}, d,{ }^{3} J=8 \mathrm{~Hz}, \mathrm{Ar}\right), 4.82\left(1 \mathrm{H}, d,{ }^{2} J=17 \mathrm{~Hz}\right.$, $\left.\mathrm{OCH}_{2}\right), 4.77\left(1 \mathrm{H}, d,{ }^{2} \mathrm{~J}=16.5 \mathrm{~Hz}, \mathrm{OCH}_{2}\right), 3.91\left(3 \mathrm{H}, s, \mathrm{OCH}_{3}\right)$, $4.28\left(2 \mathrm{H}, q,{ }^{3} \mathrm{~J}=7 \mathrm{~Hz},-\mathrm{CH}_{2} \mathrm{CH}_{3}\right), 1.33\left(3 \mathrm{H}, t,{ }^{3} \mathrm{~J}=7 \mathrm{~Hz}\right.$, $\left.\mathrm{CH}_{2} \mathrm{CH}_{3}\right), 3.26\left(1 \mathrm{H}, d d,{ }^{2} \mathrm{~J}=15 \mathrm{~Hz},{ }^{3} \mathrm{~J}=7.5 \mathrm{~Hz}, \mathrm{CH}_{2} \mathrm{CH}\right), 3.39$ $\left(1 \mathrm{H}, d d,{ }^{2} J=15 \mathrm{~Hz},{ }^{3} J=7.5 \mathrm{~Hz}, \mathrm{CH}_{2} \mathrm{CH}\right), 5.99\left(1 \mathrm{H}, m,{ }^{2} J_{\mathrm{PtH}}=\right.$ $\left.70 \mathrm{~Hz}, \mathrm{CH}=\mathrm{CH}_{2}\right), 4.69\left(1 \mathrm{H}, d,{ }^{3} J=8,{ }^{2} J_{\mathrm{PtH}}=70 \mathrm{~Hz}\right.$, cisalkene), $4.78\left(1 \mathrm{H}\right.$, ov, trans-alkene), $6.47\left(1 \mathrm{H}, d,{ }^{3} J=8.5 \mathrm{~Hz}\right.$, Ar of 2-aminopyridine), $6.6\left(1 \mathrm{H}, t,{ }^{3} J=6 \mathrm{~Hz}, \mathrm{Ar}\right), 7.35(1 \mathrm{H}, m$, $\left.{ }^{3} J=7.5 \mathrm{~Hz},{ }^{4} J=1.5 \mathrm{~Hz}, \mathrm{Ar}\right), 7.86\left(1 \mathrm{H}, d,{ }^{3} J=6 \mathrm{~Hz}, \mathrm{Ar}\right), 5.21$ $\left(\right.$ ov, $\mathrm{NH}_{2}$ ).

\subsection{Synthesis of $\left[\mathrm{Pt}(\right.$ Eteug- $\left.1 \mathrm{H}) \mathrm{Cl}\left(\mathrm{C}_{5} \mathrm{H}_{6} \mathrm{~N}_{2}\right)\right]$, (II)}

A solution of 2-aminopyridine $(0.22 \mathrm{mmol})$ in acetone $(2 \mathrm{ml})$ was added slowly to a mixture of $\left[\mathrm{Pt}_{2}(\text { Eteug- } 1 \mathrm{H})_{2} \mathrm{Cl}_{2}\right]$ $(0.1 \mathrm{mmol})$ and acetone/ethanol $(6 \mathrm{ml}, 1: 2 \mathrm{v} / \mathrm{v})$. After stirring for $2 \mathrm{~h}$ at room temperature, a yellow solution was obtained. A white precipitate was formed by slow evaporation of the solvent at $277-278 \mathrm{~K}$. The precipitate was collected by filtration and washed with ethanol. The product is soluble in acetone and chloroform, but only slightly soluble in ethanol and insoluble in water. The yield was $75 \%$. Single crystals suitable for X-ray diffraction were obtained from a acetone/ ethanol $(1: 1 v / v)$ solution via slow evaporation of the solvents at $277-278 \mathrm{~K}$.

\subsection{Data for $\left[\mathrm{Pt}(\right.$ Eteug-1 $\left.\mathrm{H}) \mathrm{Cl}\left(\mathrm{C}_{5} \mathrm{H}_{6} \mathrm{~N}_{2}\right)\right]$, (II)}

IR (Impack-410 Nicolet spectrometer, $\mathrm{KBr}, \mathrm{cm}^{-1}$ ): 3446, $3332\left(v_{\mathrm{NH}}\right) ; 3070,2941,2849\left(v_{\mathrm{CH}}\right) ; 1756\left(v_{\mathrm{C}=\mathrm{O}}\right) ; 1566$ (aromatic, $v_{\mathrm{C}=\mathrm{C}}, v_{\mathrm{C}=\mathrm{N}}$ ). 
${ }^{1} \mathrm{H} \quad$ NMR $(\delta$ p.p.m.; Bruker AVANCE $500 \mathrm{MHz}$, $\left.\mathrm{CD}_{3} \mathrm{COCD}_{3}\right): 6.66(1 \mathrm{H}, s, \mathrm{Ar}), 7.04\left(1 \mathrm{H}, s,{ }^{3} J_{\mathrm{PtH}}=40 \mathrm{~Hz}, \mathrm{Ar}\right)$, $4.59\left(1 \mathrm{H}, d,{ }^{2} J=16 \mathrm{~Hz}, 2 \mathrm{H}, \mathrm{OCH}_{2}\right), 4.55\left(1 \mathrm{H}, d,{ }^{2} J=16 \mathrm{~Hz}\right.$, $\left.\mathrm{OCH}_{2}\right), 3.73\left(3 \mathrm{H}, s,{ }^{3} \mathrm{~J}=7 \mathrm{~Hz}, \mathrm{OCH}_{3}\right), 4.21\left(2 \mathrm{H}, m,{ }^{3} \mathrm{~J}=7 \mathrm{~Hz}\right.$, $\left.\mathrm{CH}_{2} \mathrm{CH}_{3}\right), 1.28\left(3 \mathrm{H}, t,{ }^{3} \mathrm{~J}=7.0 \mathrm{~Hz}, \mathrm{CH}_{2} \mathrm{CH}_{3}\right), 2.65\left(1 \mathrm{H}, d,{ }^{2} \mathrm{~J}=\right.$ $\left.16.5 ;{ }^{3} J_{\mathrm{PtH}}=100 \mathrm{~Hz}, \mathrm{CH}_{2} \mathrm{CH}\right), 3.78\left(1 \mathrm{H}\right.$, ov, $\left.\mathrm{CH}_{2} \mathrm{CH}\right), 4.74$ $\left(1 \mathrm{H}, m,{ }^{2} J_{\mathrm{PtH}}=75 \mathrm{~Hz}, \mathrm{CH}=\mathrm{CH}_{2}\right), 3.72(1 \mathrm{H}, o v$, cis-alkene), $3.82\left(1 \mathrm{H}, d,{ }^{3} J=13.5 \mathrm{~Hz}\right.$, trans-alkene $), 6.85\left(1 \mathrm{H}, d,{ }^{3} J=\right.$ $8.5 \mathrm{~Hz}$, Ar of 2-aminopyridine ), $6.72(1 \mathrm{H}, m, \mathrm{Ar}), 7.56(1 \mathrm{H}, m$, Ar), $8.07\left(1 \mathrm{H}, d,{ }^{3} J=6 \mathrm{~Hz}, \mathrm{Ar}\right), 6.43\left(\mathrm{ov}, \mathrm{NH}_{2}\right)$.

\section{Refinement}

Crystal data, data collection and structure refinement details are summarized in Table 3. All $\mathrm{H}$ atoms were placed in idealized positions and refined in the riding mode, with $U_{\text {iso }}(\mathrm{H})$ values assigned as $1.2 U_{\text {eq }}$ of the parent atoms $(1.5$ times for methyl groups), and with $\mathrm{C}-\mathrm{H}$ distances of 0.95 (aromatic and $\left.=\mathrm{CH}_{2}\right), 0.98\left(\mathrm{CH}_{3}\right), 0.99\left(\mathrm{CH}_{2}\right)$ and $1.00 \AA$ $(\mathrm{CH})$, and $\mathrm{N}-\mathrm{H}$ distances of $0.88 \AA\left(\mathrm{NH}_{2}\right)$.

In (I), the central $\mathrm{C}$ atom in the $\mathrm{CH}_{2}-\mathrm{CH}=\mathrm{CH}_{2}$ fragment is disordered over two positions [population parameters = 0.614 (14) and $0.386(14)$ ] and was refined with constraints for bond lengths and anisotropic displacement parameters present in this fragment.

\section{Acknowledgements}

The authors thank VLIR-UOS for financial support and the Hercules Foundation for supporting the purchase of the diffractometer.

\section{Funding information}

Funding for this research was provided by: Vlaamse Interuniversitaire Raad (VLIR-UOS) Belgium (award No. ZEIN2014Z182); Hercules Foundation Belgium (award No. AKUL/09/0035).

\section{References}

Chabner, B. A. \& Roberts, T. G. (2005). Nat. Rev. Cancer, 5, 65-72.

Da, T. T., Chi, N. T. T., Van Meervelt, L., Mangwala, K. P. \& Dinh, N. H. (2015). Polyhedron, 85, 104-109.

Da, T. T., Chien, L. X., Chi, N. T. T., Hai, L. T. H. \& Dinh, N. H. (2012). J. Coord. Chem. 65, 131-142.

Da, T. T., Hai, L. T. H., Van Meervelt, L. \& Dinh, N. H. (2015). J. Coord. Chem. 68, 3525-3536.

Da, T. T., Kim, Y. M., Mai, T. T. C., Cuong, N. C. \& Dinh, N. H. (2010). J. Coord. Chem. 63, 473-483.

Dolomanov, O. V., Bourhis, L. J., Gildea, R. J., Howard, J. A. K. \& Puschmann, H. (2009). J. Appl. Cryst. 42, 339-341.

Groom, C. R., Bruno, I. J., Lightfoot, M. P. \& Ward, S. C. (2016). Acta Cryst. B72, 171-179.

Jadhav, B. K., Khandelwal, K. R., Ketkar, A. R. \& Pisal, S. S. (2004). Drug Dev. Ind. Pharm. 30(2), 195-203.

Johnstone, T. C., Park, G. Y. \& Lippard, S. J. (2014). Anticancer Res. 34, 471-476.

Kelland, L. (2007). Nat. Rev. Cancer, 7, 573-584.

Le Thi Hong, H., Dao Thi Bich, D., Nguyen Bich, N. \& Van Meervelt, L. (2016). Acta Cryst. E72, 912-917.

Nguyen Thi Thanh, C., Pham Van, T., Le Thi Hong, H. \& Van Meervelt, L. (2016). Acta Cryst. C72, 758-764.

Palatinus, L. \& Chapuis, G. (2007). J. Appl. Cryst. 40, 786-790.

Rigaku Oxford Diffraction (2015). CrysAlis PRO. Rigaku Oxford Diffraction, Yarnton, Oxfordshire, England.

Sheldrick, G. M. (2008). Acta Cryst. A64, 112-122.

Sheldrick, G. M. (2015). Acta Cryst. C71, 3-8.

Wilson, J. J. \& Lippard, S. J. (2014). Chem. Rev. 114, 4470-4495.

Wong, E. \& Giandomenico, C. M. (1999). Chem. Rev. 99, 2451-2466. 


\section{supporting information}

Acta Cryst. (2017). E73, 573-578 [https://doi.org/10.1107/S2056989017004285]

\section{Crystal structures of two platinum(II) complexes containing ethyl}

\section{eugenoxyacetate and 2-aminopyridine}

\section{Hai Le Thi Hong, Thao Nguyen Thu, Hien Nguyen and Luc Van Meervelt}

Computing details

For both compounds, data collection: CrysAlis PRO (Rigaku Oxford Diffraction, 2015); cell refinement: CrysAlis PRO (Rigaku Oxford Diffraction, 2015); data reduction: CrysAlis PRO (Rigaku Oxford Diffraction, 2015). Program(s) used to solve structure: SHELXS97 (Sheldrick, 2008) for (I); SUPERFLIP (Palatinus \& Chapuis, 2007) for (II). For both compounds, program(s) used to refine structure: SHELXL2014 (Sheldrick, 2015); molecular graphics: OLEX2 (Dolomanov et al., 2009); software used to prepare material for publication: OLEX2 (Dolomanov et al., 2009).

(I) trans-(2-Aminopyridine- $\kappa N$ ) dichlorido\{4-ethoxycarbonylmethoxy-3-methoxy-1-[(2,3- $\eta$ )-prop-2-en-1yl]benzene\}platinum(II)

\section{Crystal data}

$\left[\mathrm{PtCl}_{2}\left(\mathrm{C}_{5} \mathrm{H}_{6} \mathrm{~N}_{2}\right)\left(\mathrm{C}_{14} \mathrm{H}_{18} \mathrm{O}_{4}\right)\right]$

$M_{r}=610.39$

Triclinic, $P \overline{1}$

$a=8.3187(3) \AA$

$b=11.4119(3) \AA$

$c=12.1012(4) \AA$

$\alpha=70.437(3)^{\circ}$

$\beta=73.688(3)^{\circ}$

$\gamma=87.038(2)^{\circ}$

$V=1037.76(6) \AA^{3}$

$$
\begin{aligned}
& Z=2 \\
& F(000)=592 \\
& D_{\mathrm{x}}=1.953 \mathrm{Mg} \mathrm{m}^{-3} \\
& \text { Mo } K \alpha \text { radiation, } \lambda=0.71073 \AA \\
& \text { Cell parameters from } 13838 \text { reflections } \\
& \theta=2.7-29.0^{\circ} \\
& \mu=7.05 \mathrm{~mm}^{-1} \\
& T=100 \mathrm{~K} \\
& \text { Block, orange } \\
& 0.3 \times 0.3 \times 0.15 \mathrm{~mm}
\end{aligned}
$$

\section{Data collection}

Agilent SuperNova diffractometer (single source at offset, Eos detector)

Radiation source: micro-focus sealed X-ray tube, SuperNova (Mo) X-ray Source

Mirror monochromator Detector resolution: 15.9631 pixels $\mathrm{mm}^{-1}$ $\omega$ scans

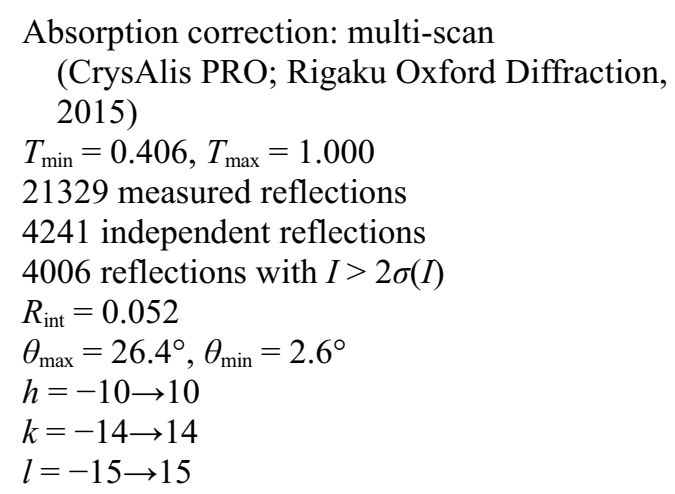




\section{Refinement}

Refinement on $F^{2}$

Least-squares matrix: full

$R\left[F^{2}>2 \sigma\left(F^{2}\right)\right]=0.029$

$w R\left(F^{2}\right)=0.065$

$S=1.04$

4241 reflections

265 parameters

26 restraints
Hydrogen site location: inferred from neighbouring sites

$\mathrm{H}$-atom parameters constrained

$w=1 /\left[\sigma^{2}\left(F_{\mathrm{o}}^{2}\right)+(0.0193 P)^{2}+4.6851 P\right]$

where $P=\left(F_{\mathrm{o}}^{2}+2 F_{\mathrm{c}}{ }^{2}\right) / 3$

$(\Delta / \sigma)_{\max }=0.001$

$\Delta \rho_{\max }=1.41 \mathrm{e} \AA^{-3}$

$\Delta \rho_{\min }=-2.25$ e $\AA^{-3}$

Special details

Geometry. All esds (except the esd in the dihedral angle between two 1.s. planes) are estimated using the full covariance matrix. The cell esds are taken into account individually in the estimation of esds in distances, angles and torsion angles; correlations between esds in cell parameters are only used when they are defined by crystal symmetry. An approximate (isotropic) treatment of cell esds is used for estimating esds involving l.s. planes.

Fractional atomic coordinates and isotropic or equivalent isotropic displacement parameters $\left(\AA^{2}\right)$

\begin{tabular}{llllll}
\hline & $x$ & $y$ & $z$ & $U_{\text {iso }} * / U_{\text {eq }}$ & Occ. $(<1)$ \\
\hline Pt1 & $0.11518(2)$ & $0.08277(2)$ & $0.62392(2)$ & $0.02167(7)$ & \\
N2 & $-0.0430(4)$ & $0.0375(3)$ & $0.7993(3)$ & $0.0155(7)$ & \\
C3 & $-0.0741(5)$ & $0.1198(4)$ & $0.8603(4)$ & $0.0146(8)$ & \\
C4 & $-0.1797(5)$ & $0.0846(4)$ & $0.9812(4)$ & $0.0170(8)$ & \\
H4 & -0.2041 & 0.1432 & 1.0232 & $0.020^{*}$ & \\
C5 & $-0.2465(5)$ & $-0.0343(4)$ & $1.0371(4)$ & $0.0205(9)$ & \\
H5 & -0.3167 & -0.0591 & 1.1187 & $0.025^{*}$ & \\
C6 & $-0.2119(6)$ & $-0.1198(4)$ & $0.9745(4)$ & $0.0208(9)$ & \\
H6 & -0.2573 & -0.2030 & 1.0124 & $0.025^{*}$ & \\
C7 & $-0.1108(5)$ & $-0.0804(4)$ & $0.8570(4)$ & $0.0175(9)$ & \\
H7 & -0.0870 & -0.1380 & 0.8139 & $0.021^{*}$ & \\
N8 & $-0.0004(4)$ & $0.2351(3)$ & $0.8045(3)$ & $0.0174(7)$ & \\
H8A & 0.0666 & 0.2556 & 0.7296 & $0.021^{*}$ & \\
H8B & -0.0193 & 0.2898 & 0.8429 & $0.021^{*}$ & \\
C19 & $-0.0885(2)$ & $0.20013(13)$ & $0.55243(12)$ & $0.0524(5)$ & \\
C110 & $0.30457(16)$ & $-0.05058(16)$ & $0.70053(13)$ & $0.0431(4)$ & \\
C11 & $0.2551(6)$ & $0.0923(4)$ & $0.4411(4)$ & $0.0277(11)$ & \\
H11A & 0.1965 & 0.1311 & 0.3822 & $0.033^{*}$ & $0.614(14)$ \\
H11B & 0.2525 & 0.0041 & 0.4747 & $0.033^{*}$ & $0.614(14)$ \\
H11C & 0.3564 & 0.0494 & 0.4378 & $0.033^{*}$ & $0.386(14)$ \\
H11D & 0.1567 & 0.0527 & 0.4423 & $0.033^{*}$ & $0.386(14)$ \\
C12A & $0.3491(9)$ & $0.1682(6)$ & $0.4797(6)$ & $0.023(2)$ & $0.614(14)$ \\
H12A & 0.4508 & 0.1263 & 0.5000 & $0.028^{*}$ & $0.614(14)$ \\
C12B & $0.2510(13)$ & $0.2166(8)$ & $0.4441(9)$ & $0.017(3)$ & $0.386(14)$ \\
H12B & 0.1698 & 0.2651 & 0.4012 & $0.020^{*}$ & $0.386(14)$ \\
C13 & $0.3685(9)$ & $0.2936(6)$ & $0.4434(6)$ & $0.063(2)$ & $0.614(14)$ \\
H13A & 0.2955 & 0.3289 & 0.3893 & $0.075^{*}$ & $0.614(14)$ \\
H13B & 0.4857 & 0.3167 & 0.3923 & $0.075^{*}$ & $0.386(14)$ \\
H13C & 0.3991 & 0.3586 & 0.3616 & $0.075^{*}$ & $0.386(14)$ \\
H13D & 0.4693 & 0.2450 & 0.4510 & $0.075^{*}$ & \\
& & & & \\
\end{tabular}




$\begin{array}{lllll}\text { C14 } & 0.3340(7) & 0.3596(5) & 0.5366(4) & 0.0345(13) \\ \text { C15 } & 0.4254(6) & 0.3359(4) & 0.6219(4) & 0.0270(11) \\ \text { H15 } & 0.5109 & 0.2775 & 0.6217 & 0.032^{*} \\ \text { C16 } & 0.3922(5) & 0.3972(4) & 0.7070(4) & 0.0175(9) \\ \text { C17 } & 0.2668(5) & 0.4837(4) & 0.7075(4) & 0.0170(8) \\ \text { C18 } & 0.1784(6) & 0.5086(4) & 0.6213(4) & 0.0243(10) \\ \text { H18 } & 0.0940 & 0.5679 & 0.6201 & 0.029^{*} \\ \text { C19 } & 0.2133(7) & 0.4467(5) & 0.5364(4) & 0.0315(12) \\ \text { H19 } & 0.1527 & 0.4649 & 0.4773 & 0.038^{*} \\ \text { O20 } & 0.4752(4) & 0.3819(3) & 0.7940(3) & 0.0220(7) \\ \text { C21 } & 0.6142(6) & 0.3034(5) & 0.7900(5) & 0.0313(12) \\ \text { H21A } & 0.6947 & 0.3334 & 0.7087 & 0.047^{*} \\ \text { H21B } & 0.5748 & 0.2180 & 0.8068 & 0.047^{*} \\ \text { H21C } & 0.6687 & 0.3047 & 0.8516 & 0.047^{*} \\ \text { O22 } & 0.2431(4) & 0.5390(3) & 0.7957(3) & 0.0178(6) \\ \text { C23 } & 0.1152(5) & 0.6250(4) & 0.8002(4) & 0.0187(9) \\ \text { H23A } & 0.1245 & 0.6827 & 0.7165 & 0.022^{*} \\ \text { H23B } & 0.1309 & 0.6750 & 0.8497 & 0.022^{*} \\ \text { C24 } & -0.0586(5) & 0.5614(4) & 0.8548(4) & 0.0163(8) \\ \text { O25 } & -0.0878(4) & 0.4502(3) & 0.9044(3) & 0.0201(6) \\ \text { O26 } & -0.1749(3) & 0.6460(3) & 0.8437(3) & 0.0170(6) \\ \text { C27 } & -0.3490(5) & 0.5976(4) & 0.9030(4) & 0.0188(9) \\ \text { H27A } & -0.3670 & 0.5590 & 0.9923 & 0.023^{*} \\ \text { H27B } & -0.3737 & 0.5333 & 0.8710 & 0.023^{*} \\ \text { C28 } & -0.4627(5) & 0.7039(4) & 0.8770(4) & 0.0192(9) \\ \text { H28A } & -0.4425 & 0.7425 & 0.7885 & 0.029^{*} \\ \text { H28B } & -0.4399 & 0.7657 & 0.9113 & 0.029^{*} \\ \text { H28C } & -0.5798 & 0.6727 & 0.9143 & 0.029^{*}\end{array}$

Atomic displacement parameters $\left(\AA^{2}\right)$

\begin{tabular}{lllllll}
\hline & $U^{11}$ & $U^{22}$ & $U^{33}$ & $U^{12}$ & $U^{13}$ & $U^{23}$ \\
\hline Pt1 & $0.03045(11)$ & $0.01466(10)$ & $0.01438(10)$ & $-0.00576(7)$ & $0.00493(7)$ & $-0.00608(7)$ \\
N2 & $0.0166(17)$ & $0.0131(17)$ & $0.0137(17)$ & $0.0016(14)$ & $-0.0029(14)$ & $-0.0015(14)$ \\
C3 & $0.0140(19)$ & $0.013(2)$ & $0.018(2)$ & $0.0038(15)$ & $-0.0069(16)$ & $-0.0051(16)$ \\
C4 & $0.016(2)$ & $0.020(2)$ & $0.015(2)$ & $0.0041(16)$ & $-0.0037(16)$ & $-0.0067(17)$ \\
C5 & $0.014(2)$ & $0.027(2)$ & $0.017(2)$ & $-0.0024(17)$ & $-0.0031(17)$ & $-0.0031(18)$ \\
C6 & $0.023(2)$ & $0.015(2)$ & $0.020(2)$ & $-0.0071(17)$ & $-0.0048(18)$ & $0.0001(17)$ \\
C7 & $0.021(2)$ & $0.015(2)$ & $0.016(2)$ & $-0.0006(17)$ & $-0.0051(17)$ & $-0.0042(17)$ \\
N8 & $0.0212(18)$ & $0.0122(17)$ & $0.0168(18)$ & $0.0005(14)$ & $-0.0011(14)$ & $-0.0054(14)$ \\
C19 & $0.1016(13)$ & $0.0365(8)$ & $0.0210(6)$ & $0.0429(8)$ & $-0.0248(7)$ & $-0.0113(6)$ \\
C110 & $0.0236(6)$ & $0.0824(11)$ & $0.0382(7)$ & $0.0192(7)$ & $-0.0099(5)$ & $-0.0404(8)$ \\
C11 & $0.038(3)$ & $0.022(2)$ & $0.014(2)$ & $-0.007(2)$ & $0.0094(19)$ & $-0.0080(18)$ \\
C12A & $0.020(4)$ & $0.024(4)$ & $0.016(4)$ & $-0.007(3)$ & $0.009(3)$ & $-0.006(3)$ \\
C12B & $0.024(6)$ & $0.020(5)$ & $0.005(5)$ & $-0.001(4)$ & $-0.003(4)$ & $-0.004(4)$ \\
C13 & $0.071(4)$ & $0.072(4)$ & $0.038(3)$ & $-0.051(4)$ & $0.030(3)$ & $-0.040(3)$ \\
C14 & $0.040(3)$ & $0.039(3)$ & $0.018(2)$ & $-0.031(3)$ & $0.015(2)$ & $-0.015(2)$ \\
C15 & $0.022(2)$ & $0.023(2)$ & $0.028(3)$ & $-0.0113(19)$ & $0.0111(19)$ & $-0.012(2)$
\end{tabular}




$\begin{array}{lllllll}\mathrm{C} 16 & 0.016(2) & 0.013(2) & 0.019(2) & -0.0036(16) & -0.0001(17) & -0.0037(17) \\ \mathrm{C} 17 & 0.015(2) & 0.017(2) & 0.015(2) & -0.0030(16) & -0.0003(16) & -0.0024(17) \\ \mathrm{C} 18 & 0.024(2) & 0.022(2) & 0.020(2) & -0.0099(19) & -0.0062(19) & 0.0024(19) \\ \mathrm{C} 19 & 0.038(3) & 0.036(3) & 0.014(2) & -0.023(2) & -0.004(2) & 0.001(2) \\ \mathrm{O} 20 & 0.0160(15) & 0.0223(16) & 0.0280(17) & 0.0099(13) & -0.0059(13) & -0.0104(14) \\ \mathrm{C} 21 & 0.020(2) & 0.028(3) & 0.038(3) & 0.012(2) & -0.001(2) & -0.007(2) \\ \mathrm{O} 22 & 0.0159(14) & 0.0168(15) & 0.0246(16) & 0.0060(12) & -0.0075(12) & -0.0112(13) \\ \mathrm{C} 23 & 0.016(2) & 0.012(2) & 0.028(2) & 0.0032(16) & -0.0051(18) & -0.0079(18) \\ \mathrm{C} 24 & 0.018(2) & 0.015(2) & 0.016(2) & 0.0015(16) & -0.0059(16) & -0.0042(17) \\ \mathrm{O} 25 & 0.0216(16) & 0.0144(15) & 0.0222(16) & -0.0002(12) & -0.0033(13) & -0.0057(13) \\ \mathrm{O} 26 & 0.0130(14) & 0.0128(14) & 0.0220(16) & 0.0001(11) & -0.0015(12) & -0.0044(12) \\ \mathrm{C} 27 & 0.013(2) & 0.019(2) & 0.021(2) & -0.0030(17) & 0.0005(17) & -0.0057(18) \\ \mathrm{C} 28 & 0.015(2) & 0.024(2) & 0.017(2) & 0.0017(17) & -0.0038(17) & -0.0062(18) \\ \end{array}$

Geometric parameters $\left(\AA,{ }^{\circ}\right)$

\begin{tabular}{|c|c|c|c|}
\hline $\mathrm{Pt} 1-\mathrm{N} 2$ & $2.066(3)$ & $\mathrm{C} 13-\mathrm{H} 13 \mathrm{C}$ & 0.9900 \\
\hline $\mathrm{Pt} 1-\mathrm{C} 19$ & $2.2860(14)$ & $\mathrm{C} 13-\mathrm{H} 13 \mathrm{D}$ & 0.9900 \\
\hline $\mathrm{Pt} 1-\mathrm{Cl} 10$ & $2.2990(14)$ & $\mathrm{C} 13-\mathrm{C} 14$ & $1.513(7)$ \\
\hline $\mathrm{Pt} 1-\mathrm{C} 11$ & $2.161(4)$ & $\mathrm{C} 14-\mathrm{C} 15$ & $1.395(8)$ \\
\hline $\mathrm{Pt} 1-\mathrm{C} 12 \mathrm{~A}$ & $2.221(7)$ & $\mathrm{C} 14-\mathrm{C} 19$ & $1.376(8)$ \\
\hline $\mathrm{Pt} 1-\mathrm{C} 12 \mathrm{~B}$ & $2.217(10)$ & $\mathrm{C} 15-\mathrm{H} 15$ & 0.9500 \\
\hline $\mathrm{N} 2-\mathrm{C} 3$ & $1.352(5)$ & $\mathrm{C} 15-\mathrm{C} 16$ & $1.389(6)$ \\
\hline $\mathrm{N} 2-\mathrm{C} 7$ & $1.358(5)$ & $\mathrm{C} 16-\mathrm{C} 17$ & $1.399(6)$ \\
\hline $\mathrm{C} 3-\mathrm{C} 4$ & $1.412(6)$ & $\mathrm{C} 16-\mathrm{O} 20$ & $1.375(5)$ \\
\hline $\mathrm{C} 3-\mathrm{N} 8$ & $1.348(5)$ & $\mathrm{C} 17-\mathrm{C} 18$ & $1.386(6)$ \\
\hline $\mathrm{C} 4-\mathrm{H} 4$ & 0.9500 & $\mathrm{C} 17-\mathrm{O} 22$ & $1.377(5)$ \\
\hline $\mathrm{C} 4-\mathrm{C} 5$ & $1.364(6)$ & $\mathrm{C} 18-\mathrm{H} 18$ & 0.9500 \\
\hline $\mathrm{C} 5-\mathrm{H} 5$ & 0.9500 & $\mathrm{C} 18-\mathrm{C} 19$ & $1.391(7)$ \\
\hline $\mathrm{C} 5-\mathrm{C} 6$ & $1.397(6)$ & $\mathrm{C} 19-\mathrm{H} 19$ & 0.9500 \\
\hline $\mathrm{C} 6-\mathrm{H} 6$ & 0.9500 & $\mathrm{O} 20-\mathrm{C} 21$ & $1.426(5)$ \\
\hline $\mathrm{C} 6-\mathrm{C} 7$ & $1.367(6)$ & $\mathrm{C} 21-\mathrm{H} 21 \mathrm{~A}$ & 0.9800 \\
\hline $\mathrm{C} 7-\mathrm{H} 7$ & 0.9500 & $\mathrm{C} 21-\mathrm{H} 21 \mathrm{~B}$ & 0.9800 \\
\hline N8-H8A & 0.8800 & $\mathrm{C} 21-\mathrm{H} 21 \mathrm{C}$ & 0.9800 \\
\hline $\mathrm{N} 8-\mathrm{H} 8 \mathrm{~B}$ & 0.8800 & $\mathrm{O} 22-\mathrm{C} 23$ & $1.413(5)$ \\
\hline $\mathrm{C} 11-\mathrm{H} 11 \mathrm{~A}$ & 0.9500 & $\mathrm{C} 23-\mathrm{H} 23 \mathrm{~A}$ & 0.9900 \\
\hline $\mathrm{C} 11-\mathrm{H} 11 \mathrm{~B}$ & 0.9500 & $\mathrm{C} 23-\mathrm{H} 23 \mathrm{~B}$ & 0.9900 \\
\hline $\mathrm{C} 11-\mathrm{H} 11 \mathrm{C}$ & 0.9500 & $\mathrm{C} 23-\mathrm{C} 24$ & $1.518(6)$ \\
\hline $\mathrm{C} 11-\mathrm{H} 11 \mathrm{D}$ & 0.9500 & $\mathrm{C} 24-\mathrm{O} 25$ & $1.211(5)$ \\
\hline $\mathrm{C} 11-\mathrm{C} 12 \mathrm{~A}$ & $1.458(8)$ & $\mathrm{C} 24-\mathrm{O} 26$ & $1.333(5)$ \\
\hline $\mathrm{C} 11-\mathrm{C} 12 \mathrm{~B}$ & $1.429(9)$ & $\mathrm{O} 26-\mathrm{C} 27$ & $1.467(5)$ \\
\hline $\mathrm{C} 12 \mathrm{~A}-\mathrm{H} 12 \mathrm{~A}$ & 1.0000 & $\mathrm{C} 27-\mathrm{H} 27 \mathrm{~A}$ & 0.9900 \\
\hline $\mathrm{C} 12 \mathrm{~A}-\mathrm{C} 13$ & $1.353(9)$ & $\mathrm{C} 27-\mathrm{H} 27 \mathrm{~B}$ & 0.9900 \\
\hline $\mathrm{C} 12 \mathrm{~B}-\mathrm{H} 12 \mathrm{~B}$ & 1.0000 & $\mathrm{C} 27-\mathrm{C} 28$ & $1.502(6)$ \\
\hline $\mathrm{C} 12 \mathrm{~B}-\mathrm{C} 13$ & $1.344(9)$ & $\mathrm{C} 28-\mathrm{H} 28 \mathrm{~A}$ & 0.9800 \\
\hline $\mathrm{C} 13-\mathrm{H} 13 \mathrm{~A}$ & 0.9900 & $\mathrm{C} 28-\mathrm{H} 28 \mathrm{~B}$ & 0.9800 \\
\hline $\mathrm{C} 13-\mathrm{H} 13 \mathrm{~B}$ & 0.9900 & $\mathrm{C} 28-\mathrm{H} 28 \mathrm{C}$ & 0.9800 \\
\hline
\end{tabular}




\begin{tabular}{|c|c|c|c|}
\hline $\mathrm{N} 2-\mathrm{Pt} 1-\mathrm{Cl} 9$ & $89.18(10)$ & $\mathrm{C} 12 \mathrm{~A}-\mathrm{C} 13-\mathrm{H} 13 \mathrm{~B}$ & 107.2 \\
\hline $\mathrm{N} 2-\mathrm{Pt} 1-\mathrm{Cl} 10$ & $88.85(10)$ & $\mathrm{C} 12 \mathrm{~A}-\mathrm{C} 13-\mathrm{C} 14$ & $120.4(6)$ \\
\hline $\mathrm{N} 2-\mathrm{Pt} 1-\mathrm{C} 11$ & $167.04(16)$ & $\mathrm{C} 12 \mathrm{~B}-\mathrm{C} 13-\mathrm{H} 13 \mathrm{C}$ & 107.2 \\
\hline $\mathrm{N} 2-\mathrm{Pt} 1-\mathrm{C} 12 \mathrm{~A}$ & $153.0(2)$ & $\mathrm{C} 12 \mathrm{~B}-\mathrm{C} 13-\mathrm{H} 13 \mathrm{D}$ & 107.2 \\
\hline $\mathrm{N} 2-\mathrm{Pt} 1-\mathrm{C} 12 \mathrm{~B}$ & $153.2(3)$ & $\mathrm{C} 12 \mathrm{~B}-\mathrm{C} 13-\mathrm{C} 14$ & $120.7(6)$ \\
\hline $\mathrm{C} 19-\mathrm{Pt} 1-\mathrm{Cl10}$ & $174.90(6)$ & $\mathrm{H} 13 \mathrm{~A}-\mathrm{C} 13-\mathrm{H} 13 \mathrm{~B}$ & 106.8 \\
\hline $\mathrm{C} 11-\mathrm{Pt} 1-\mathrm{C} 19$ & $91.10(15)$ & $\mathrm{H} 13 \mathrm{C}-\mathrm{C} 13-\mathrm{H} 13 \mathrm{D}$ & 106.8 \\
\hline $\mathrm{C} 11-\mathrm{Pt} 1-\mathrm{Cl} 10$ & 89.77 (15) & $\mathrm{C} 14-\mathrm{C} 13-\mathrm{H} 13 \mathrm{~A}$ & 107.2 \\
\hline $\mathrm{C} 11-\mathrm{Pt} 1-\mathrm{C} 12 \mathrm{~A}$ & $38.8(2)$ & $\mathrm{C} 14-\mathrm{C} 13-\mathrm{H} 13 \mathrm{~B}$ & 107.2 \\
\hline $\mathrm{C} 11-\mathrm{Pt} 1-\mathrm{C} 12 \mathrm{~B}$ & $38.1(2)$ & $\mathrm{C} 14-\mathrm{C} 13-\mathrm{H} 13 \mathrm{C}$ & 107.2 \\
\hline $\mathrm{C} 12 \mathrm{~A}-\mathrm{Pt} 1-\mathrm{Cl} 9$ & $103.0(2)$ & $\mathrm{C} 14-\mathrm{C} 13-\mathrm{H} 13 \mathrm{D}$ & 107.2 \\
\hline $\mathrm{C} 12 \mathrm{~A}-\mathrm{Pt} 1-\mathrm{C} 110$ & $80.8(2)$ & $\mathrm{C} 15-\mathrm{C} 14-\mathrm{C} 13$ & $121.1(6)$ \\
\hline $\mathrm{C} 12 \mathrm{~B}-\mathrm{Pt} 1-\mathrm{Cl} 9$ & $75.1(3)$ & $\mathrm{C} 19-\mathrm{C} 14-\mathrm{C} 13$ & $119.9(6)$ \\
\hline $\mathrm{C} 12 \mathrm{~B}-\mathrm{Pt} 1-\mathrm{C} 110$ & $108.4(3)$ & $\mathrm{C} 19-\mathrm{C} 14-\mathrm{C} 15$ & $119.0(5)$ \\
\hline $\mathrm{C} 3-\mathrm{N} 2-\mathrm{Pt} 1$ & $122.0(3)$ & $\mathrm{C} 14-\mathrm{C} 15-\mathrm{H} 15$ & 119.8 \\
\hline $\mathrm{C} 3-\mathrm{N} 2-\mathrm{C} 7$ & $119.1(4)$ & $\mathrm{C} 16-\mathrm{C} 15-\mathrm{C} 14$ & $120.5(5)$ \\
\hline $\mathrm{C} 7-\mathrm{N} 2-\mathrm{Pt} 1$ & $118.9(3)$ & $\mathrm{C} 16-\mathrm{C} 15-\mathrm{H} 15$ & 119.8 \\
\hline $\mathrm{N} 2-\mathrm{C} 3-\mathrm{C} 4$ & $120.4(4)$ & $\mathrm{C} 15-\mathrm{C} 16-\mathrm{C} 17$ & $120.0(4)$ \\
\hline $\mathrm{N} 8-\mathrm{C} 3-\mathrm{N} 2$ & $118.8(4)$ & $\mathrm{O} 20-\mathrm{C} 16-\mathrm{C} 15$ & $125.3(4)$ \\
\hline $\mathrm{N} 8-\mathrm{C} 3-\mathrm{C} 4$ & $120.8(4)$ & $\mathrm{O} 20-\mathrm{C} 16-\mathrm{C} 17$ & $114.6(4)$ \\
\hline $\mathrm{C} 3-\mathrm{C} 4-\mathrm{H} 4$ & 120.3 & $\mathrm{C} 18-\mathrm{C} 17-\mathrm{C} 16$ & $119.3(4)$ \\
\hline $\mathrm{C} 5-\mathrm{C} 4-\mathrm{C} 3$ & $119.4(4)$ & $\mathrm{O} 22-\mathrm{C} 17-\mathrm{C} 16$ & $115.6(4)$ \\
\hline $\mathrm{C} 5-\mathrm{C} 4-\mathrm{H} 4$ & 120.3 & $\mathrm{O} 22-\mathrm{C} 17-\mathrm{C} 18$ & $125.1(4)$ \\
\hline $\mathrm{C} 4-\mathrm{C} 5-\mathrm{H} 5$ & 119.9 & $\mathrm{C} 17-\mathrm{C} 18-\mathrm{H} 18$ & 120.0 \\
\hline $\mathrm{C} 4-\mathrm{C} 5-\mathrm{C} 6$ & $120.1(4)$ & $\mathrm{C} 17-\mathrm{C} 18-\mathrm{C} 19$ & $120.0(5)$ \\
\hline $\mathrm{C} 6-\mathrm{C} 5-\mathrm{H} 5$ & 119.9 & $\mathrm{C} 19-\mathrm{C} 18-\mathrm{H} 18$ & 120.0 \\
\hline $\mathrm{C} 5-\mathrm{C} 6-\mathrm{H} 6$ & 120.9 & $\mathrm{C} 14-\mathrm{C} 19-\mathrm{C} 18$ & $121.2(5)$ \\
\hline $\mathrm{C} 7-\mathrm{C} 6-\mathrm{C} 5$ & $118.1(4)$ & $\mathrm{C} 14-\mathrm{C} 19-\mathrm{H} 19$ & 119.4 \\
\hline $\mathrm{C} 7-\mathrm{C} 6-\mathrm{H} 6$ & 120.9 & $\mathrm{C} 18-\mathrm{C} 19-\mathrm{H} 19$ & 119.4 \\
\hline $\mathrm{N} 2-\mathrm{C} 7-\mathrm{C} 6$ & $122.9(4)$ & $\mathrm{C} 16-\mathrm{O} 20-\mathrm{C} 21$ & $116.7(4)$ \\
\hline $\mathrm{N} 2-\mathrm{C} 7-\mathrm{H} 7$ & 118.5 & $\mathrm{O} 20-\mathrm{C} 21-\mathrm{H} 21 \mathrm{~A}$ & 109.5 \\
\hline $\mathrm{C} 6-\mathrm{C} 7-\mathrm{H} 7$ & 118.5 & $\mathrm{O} 20-\mathrm{C} 21-\mathrm{H} 21 \mathrm{~B}$ & 109.5 \\
\hline $\mathrm{C} 3-\mathrm{N} 8-\mathrm{H} 8 \mathrm{~A}$ & 120.0 & $\mathrm{O} 20-\mathrm{C} 21-\mathrm{H} 21 \mathrm{C}$ & 109.5 \\
\hline $\mathrm{C} 3-\mathrm{N} 8-\mathrm{H} 8 \mathrm{~B}$ & 120.0 & $\mathrm{H} 21 \mathrm{~A}-\mathrm{C} 21-\mathrm{H} 21 \mathrm{~B}$ & 109.5 \\
\hline $\mathrm{H} 8 \mathrm{~A}-\mathrm{N} 8-\mathrm{H} 8 \mathrm{~B}$ & 120.0 & $\mathrm{H} 21 \mathrm{~A}-\mathrm{C} 21-\mathrm{H} 21 \mathrm{C}$ & 109.5 \\
\hline $\mathrm{Pt} 1-\mathrm{C} 11-\mathrm{H} 11 \mathrm{~A}$ & 112.8 & $\mathrm{H} 21 \mathrm{~B}-\mathrm{C} 21-\mathrm{H} 21 \mathrm{C}$ & 109.5 \\
\hline $\mathrm{Pt} 1-\mathrm{C} 11-\mathrm{H} 11 \mathrm{~B}$ & 84.7 & $\mathrm{C} 17-\mathrm{O} 22-\mathrm{C} 23$ & $117.0(3)$ \\
\hline $\mathrm{Pt} 1-\mathrm{C} 11-\mathrm{H} 11 \mathrm{C}$ & 113.4 & $\mathrm{O} 22-\mathrm{C} 23-\mathrm{H} 23 \mathrm{~A}$ & 109.2 \\
\hline Pt1-C11-H11D & 83.9 & $\mathrm{O} 22-\mathrm{C} 23-\mathrm{H} 23 \mathrm{~B}$ & 109.2 \\
\hline $\mathrm{H} 11 \mathrm{~A}-\mathrm{C} 11-\mathrm{H} 11 \mathrm{~B}$ & 120.0 & $\mathrm{O} 22-\mathrm{C} 23-\mathrm{C} 24$ & $112.2(3)$ \\
\hline $\mathrm{H} 11 \mathrm{C}-\mathrm{C} 11-\mathrm{H} 11 \mathrm{D}$ & 120.0 & $\mathrm{H} 23 \mathrm{~A}-\mathrm{C} 23-\mathrm{H} 23 \mathrm{~B}$ & 107.9 \\
\hline $\mathrm{C} 12 \mathrm{~A}-\mathrm{C} 11-\mathrm{Pt} 1$ & $72.8(3)$ & $\mathrm{C} 24-\mathrm{C} 23-\mathrm{H} 23 \mathrm{~A}$ & 109.2 \\
\hline $\mathrm{C} 12 \mathrm{~A}-\mathrm{C} 11-\mathrm{H} 11 \mathrm{~A}$ & 120.0 & $\mathrm{C} 24-\mathrm{C} 23-\mathrm{H} 23 \mathrm{~B}$ & 109.2 \\
\hline $\mathrm{C} 12 \mathrm{~A}-\mathrm{C} 11-\mathrm{H} 11 \mathrm{~B}$ & 120.0 & $\mathrm{O} 25-\mathrm{C} 24-\mathrm{C} 23$ & $125.1(4)$ \\
\hline $\mathrm{C} 12 \mathrm{~B}-\mathrm{C} 11-\mathrm{Pt} 1$ & $73.1(4)$ & $\mathrm{O} 25-\mathrm{C} 24-\mathrm{O} 26$ & $124.8(4)$ \\
\hline $\mathrm{C} 12 \mathrm{~B}-\mathrm{C} 11-\mathrm{H} 11 \mathrm{C}$ & 120.0 & $\mathrm{O} 26-\mathrm{C} 24-\mathrm{C} 23$ & $110.1(3)$ \\
\hline $\mathrm{C} 12 \mathrm{~B}-\mathrm{C} 11-\mathrm{H} 11 \mathrm{D}$ & 120.0 & $\mathrm{C} 24-\mathrm{O} 26-\mathrm{C} 27$ & $115.6(3)$ \\
\hline $\mathrm{Pt} 1-\mathrm{C} 12 \mathrm{~A}-\mathrm{H} 12 \mathrm{~A}$ & 111.8 & $\mathrm{O} 26-\mathrm{C} 27-\mathrm{H} 27 \mathrm{~A}$ & 110.0 \\
\hline
\end{tabular}




\begin{tabular}{|c|c|}
\hline $\mathrm{C} 11-\mathrm{C} 12 \mathrm{~A}-\mathrm{Pt} 1$ & $68.3(3)$ \\
\hline $\mathrm{C} 11-\mathrm{C} 12 \mathrm{~A}-\mathrm{H} 12 \mathrm{~A}$ & 111.8 \\
\hline $\mathrm{C} 13-\mathrm{C} 12 \mathrm{~A}-\mathrm{Pt} 1$ & $116.3(5)$ \\
\hline $\mathrm{C} 13-\mathrm{C} 12 \mathrm{~A}-\mathrm{C} 11$ & $129.2(6)$ \\
\hline $\mathrm{C} 13-\mathrm{C} 12 \mathrm{~A}-\mathrm{H} 12 \mathrm{~A}$ & 111.8 \\
\hline $\mathrm{Pt} 1-\mathrm{C} 12 \mathrm{~B}-\mathrm{H} 12 \mathrm{~B}$ & 110.3 \\
\hline $\mathrm{C} 11-\mathrm{C} 12 \mathrm{~B}-\mathrm{Pt} 1$ & $68.8(4)$ \\
\hline $\mathrm{C} 11-\mathrm{C} 12 \mathrm{~B}-\mathrm{H} 12 \mathrm{~B}$ & 110.3 \\
\hline $\mathrm{C} 13-\mathrm{C} 12 \mathrm{~B}-\mathrm{Pt} 1$ & $116.9(6)$ \\
\hline $\mathrm{C} 13-\mathrm{C} 12 \mathrm{~B}-\mathrm{C} 11$ & $132.6(8)$ \\
\hline $\mathrm{C} 13-\mathrm{C} 12 \mathrm{~B}-\mathrm{H} 12 \mathrm{~B}$ & 110.3 \\
\hline $\mathrm{C} 12 \mathrm{~A}-\mathrm{C} 13-\mathrm{H} 13 \mathrm{~A}$ & 107.2 \\
\hline $\mathrm{Pt} 1-\mathrm{N} 2-\mathrm{C} 3-\mathrm{C} 4$ & $-178.4(3)$ \\
\hline $\mathrm{Pt} 1-\mathrm{N} 2-\mathrm{C} 3-\mathrm{N} 8$ & $0.5(5)$ \\
\hline $\mathrm{Pt} 1-\mathrm{N} 2-\mathrm{C} 7-\mathrm{C} 6$ & $177.6(3)$ \\
\hline $\mathrm{Pt} 1-\mathrm{C} 11-\mathrm{C} 12 \mathrm{~A}-\mathrm{C} 13$ & $-106.9(7)$ \\
\hline $\mathrm{Pt} 1-\mathrm{C} 11-\mathrm{C} 12 \mathrm{~B}-\mathrm{C} 13$ & $107.7(11)$ \\
\hline $\mathrm{Pt} 1-\mathrm{C} 12 \mathrm{~A}-\mathrm{C} 13-\mathrm{C} 14$ & $46.1(8)$ \\
\hline $\mathrm{Pt} 1-\mathrm{C} 12 \mathrm{~B}-\mathrm{C} 13-\mathrm{C} 14$ & $-44.9(10)$ \\
\hline $\mathrm{N} 2-\mathrm{C} 3-\mathrm{C} 4-\mathrm{C} 5$ & $1.8(6)$ \\
\hline $\mathrm{C} 3-\mathrm{N} 2-\mathrm{C} 7-\mathrm{C} 6$ & $1.0(6)$ \\
\hline $\mathrm{C} 3-\mathrm{C} 4-\mathrm{C} 5-\mathrm{C} 6$ & $-0.7(6)$ \\
\hline $\mathrm{C} 4-\mathrm{C} 5-\mathrm{C} 6-\mathrm{C} 7$ & $-0.2(6)$ \\
\hline $\mathrm{C} 5-\mathrm{C} 6-\mathrm{C} 7-\mathrm{N} 2$ & $0.1(7)$ \\
\hline $\mathrm{C} 7-\mathrm{N} 2-\mathrm{C} 3-\mathrm{C} 4$ & $-1.9(6)$ \\
\hline $\mathrm{C} 7-\mathrm{N} 2-\mathrm{C} 3-\mathrm{N} 8$ & $177.0(4)$ \\
\hline $\mathrm{N} 8-\mathrm{C} 3-\mathrm{C} 4-\mathrm{C} 5$ & $-177.1(4)$ \\
\hline $\mathrm{C} 11-\mathrm{C} 12 \mathrm{~A}-\mathrm{C} 13-\mathrm{C} 14$ & $128.7(7)$ \\
\hline $\mathrm{C} 11-\mathrm{C} 12 \mathrm{~B}-\mathrm{C} 13-\mathrm{C} 14$ & $-130.1(10)$ \\
\hline $\mathrm{C} 12 \mathrm{~A}-\mathrm{C} 13-\mathrm{C} 14-\mathrm{C} 15$ & $61.2(8)$ \\
\hline $\mathrm{C} 12 \mathrm{~A}-\mathrm{C} 13-\mathrm{C} 14-\mathrm{C} 19$ & $-120.3(7)$ \\
\hline $\mathrm{C} 12 \mathrm{~B}-\mathrm{C} 13-\mathrm{C} 14-\mathrm{C} 15$ & $116.1(8)$ \\
\hline $\mathrm{C} 12 \mathrm{~B}-\mathrm{C} 13-\mathrm{C} 14-\mathrm{C} 19$ & $-65.4(9)$ \\
\hline $\mathrm{C} 13-\mathrm{C} 14-\mathrm{C} 15-\mathrm{C} 16$ & $-180.0(4)$ \\
\hline
\end{tabular}

$\begin{array}{ll}\mathrm{O} 26-\mathrm{C} 27-\mathrm{H} 27 \mathrm{~B} & 110.0 \\ \mathrm{O} 26-\mathrm{C} 27-\mathrm{C} 28 & 108.4(3) \\ \mathrm{H} 27 \mathrm{~A}-\mathrm{C} 27-\mathrm{H} 27 \mathrm{~B} & 108.4 \\ \mathrm{C} 28-\mathrm{C} 27-\mathrm{H} 27 \mathrm{~A} & 110.0 \\ \mathrm{C} 28-\mathrm{C} 27-\mathrm{H} 27 \mathrm{~B} & 110.0 \\ \mathrm{C} 27-\mathrm{C} 28-\mathrm{H} 28 \mathrm{~A} & 109.5 \\ \mathrm{C} 27-\mathrm{C} 28-\mathrm{H} 28 \mathrm{~B} & 109.5 \\ \mathrm{C} 27-\mathrm{C} 28-\mathrm{H} 28 \mathrm{C} & 109.5 \\ \mathrm{H} 28 \mathrm{~A}-\mathrm{C} 28-\mathrm{H} 28 \mathrm{~B} & 109.5 \\ \mathrm{H} 28 \mathrm{~A}-\mathrm{C} 28-\mathrm{H} 28 \mathrm{C} & 109.5 \\ \mathrm{H} 28 \mathrm{~B}-\mathrm{C} 28-\mathrm{H} 28 \mathrm{C} & 109.5\end{array}$

$179.8(4)$

$-0.2(6)$

$-179.4(4)$

$-1.7(7)$

$\mathrm{C} 15-\mathrm{C} 14-\mathrm{C} 19-\mathrm{C} 18$

$-0.9(6)$

$\mathrm{C} 15-\mathrm{C} 16-\mathrm{C} 17-\mathrm{C} 18$

179.7 (4)

$\mathrm{C} 15-\mathrm{C} 16-\mathrm{C} 17-\mathrm{O} 22$

$4.8(6)$

$\mathrm{C} 15-\mathrm{C} 16-\mathrm{O} 20-\mathrm{C} 21$

$0.8(6)$

$\mathrm{C} 16-\mathrm{C} 17-\mathrm{O} 22-\mathrm{C} 23$

$-178.8(4)$

$-174.4(4)$

$\mathrm{C} 17-\mathrm{C} 16-\mathrm{O} 20-\mathrm{C} 21$

$0.5(7)$

$\mathrm{C} 17-\mathrm{C} 18-\mathrm{C} 19-\mathrm{C} 14$

$74.6(5)$

$1.9(6)$

$\mathrm{C} 18-\mathrm{C} 17-\mathrm{O} 22-\mathrm{C} 23$

$1.5(7)$

$\mathrm{C} 19-\mathrm{C} 14-\mathrm{C} 15-\mathrm{C} 16$

$178.4(4)$

$\mathrm{O} 20-\mathrm{C} 16-\mathrm{C} 17-\mathrm{C} 18$

$-1.0(5)$

$\mathrm{O} 22-\mathrm{C} 17-\mathrm{C} 18-\mathrm{C} 19$

$-179.9(4)$

$\mathrm{O} 22-\mathrm{C} 23-\mathrm{C} 24-\mathrm{O} 25$

$9.5(6)$

$\mathrm{O} 22-\mathrm{C} 23-\mathrm{C} 24-\mathrm{O} 26$

$-172.5(3)$

$\mathrm{C} 23-\mathrm{C} 24-\mathrm{O} 26-\mathrm{C} 27$

$-174.6(3)$

$\mathrm{C} 24-\mathrm{O} 26-\mathrm{C} 27-\mathrm{C} 28$

$-177.6(3)$

$\mathrm{O} 25-\mathrm{C} 24-\mathrm{O} 26-\mathrm{C} 27$

3.5 (6)

Hydrogen-bond geometry $(\AA, \stackrel{\circ}{)}$

$\mathrm{Cg} 1$ is the centroid of the $\mathrm{C} 14-\mathrm{C} 19$ ring.

\begin{tabular}{lllll}
\hline$D-\mathrm{H} \cdots A$ & $D-\mathrm{H}$ & $\mathrm{H} \cdots A$ & $D \cdots A$ & $D-\mathrm{H} \cdots A$ \\
\hline $\mathrm{N} 8-\mathrm{H} 8 B^{\cdots} \cdots \mathrm{O} 25$ & 0.88 & 2.19 & $3.054(5)$ & 167 \\
$\mathrm{C} 7-\mathrm{H} 7 \cdots \mathrm{O} 26^{\mathrm{i}}$ & 0.95 & 2.49 & $3.258(6)$ & 138 \\
$\mathrm{C} 18-\mathrm{H} 18 \cdots \mathrm{C} 9^{\mathrm{ii}}$ & 0.95 & 2.78 & $3.460(5)$ & 130 \\
$\mathrm{~N} 8-\mathrm{H} 8 A^{\mathrm{ii}} \mathrm{Cg} 1$ & 0.88 & 2.53 & $3.166(4)$ & 129 \\
\hline
\end{tabular}

Symmetry codes: (i) $x, y-1, z$; (ii) $-x,-y+1,-z+1$. 
(II) (2-Aminopyridine- $\kappa N)$ chlorido\{5-ethoxycarbonylmethoxy-4-methoxy-1-[(2,3- $\eta)$-prop-2-en-1-yl]phenyl-

$\left.\kappa C^{1}\right\}$ platinum(II)

Crystal data

$\left[\mathrm{Pt}\left(\mathrm{C}_{14} \mathrm{H}_{17} \mathrm{O}_{4}\right) \mathrm{Cl}\left(\mathrm{C}_{5} \mathrm{H}_{6} \mathrm{~N}_{2}\right)\right]$

$M_{r}=573.93$

Monoclinic, $P 2_{1} / c$

$a=15.1198(5) \AA$

$b=10.0855(2) \AA$

$c=14.1855$ (4) $\AA$

$\beta=117.329(4)^{\circ}$

$V=1921.72(11) \AA^{3}$

$Z=4$

$F(000)=1112$

$D_{\mathrm{x}}=1.984 \mathrm{Mg} \mathrm{m}^{-3}$

Mo $K \alpha$ radiation, $\lambda=0.71073 \AA$

Cell parameters from 14326 reflections

$\theta=3.2-29.1^{\circ}$

$\mu=7.47 \mathrm{~mm}^{-1}$

$T=100 \mathrm{~K}$

Block, white

$0.3 \times 0.3 \times 0.2 \mathrm{~mm}$

\section{Data collection}

Agilent SuperNova

diffractometer (single source at offset, Eos detector)

Radiation source: micro-focus sealed X-ray tube, SuperNova (Mo) X-ray Source

Mirror monochromator

Detector resolution: 15.9631 pixels $\mathrm{mm}^{-1}$

$\omega$ scans

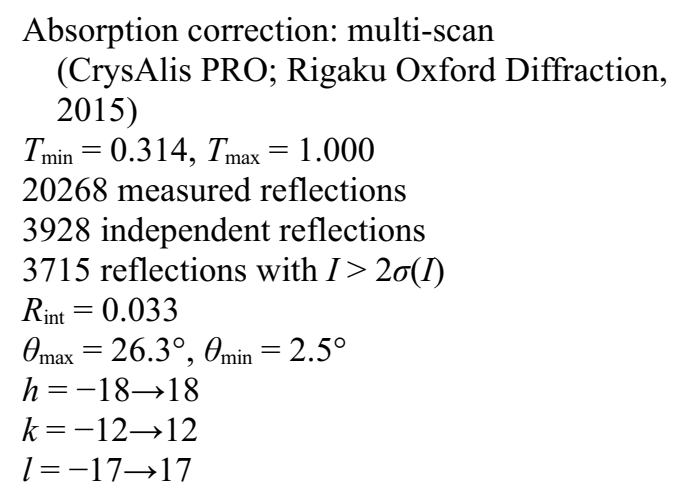

Refinement

Refinement on $F^{2}$

Least-squares matrix: full

Hydrogen site location: inferred from neighbouring sites

$R\left[F^{2}>2 \sigma\left(F^{2}\right)\right]=0.016$

$w R\left(F^{2}\right)=0.038$

$S=1.06$

3928 reflections

246 parameters

0 restraints

$\mathrm{H}$-atom parameters constrained

$w=1 /\left[\sigma^{2}\left(F_{\mathrm{o}}^{2}\right)+(0.0159 P)^{2}+1.3858 P\right]$

where $P=\left(F_{\mathrm{o}}^{2}+2 F_{\mathrm{c}}^{2}\right) / 3$

$(\Delta / \sigma)_{\max }<0.001$

$\Delta \rho_{\max }=0.69 \mathrm{e} \AA^{-3}$

$\Delta \rho_{\min }=-1.04$ e $\AA^{-3}$

Special details

Geometry. All esds (except the esd in the dihedral angle between two 1.s. planes) are estimated using the full covariance matrix. The cell esds are taken into account individually in the estimation of esds in distances, angles and torsion angles; correlations between esds in cell parameters are only used when they are defined by crystal symmetry. An approximate (isotropic) treatment of cell esds is used for estimating esds involving l.s. planes.

Fractional atomic coordinates and isotropic or equivalent isotropic displacement parameters $\left(\AA^{2}\right)$

\begin{tabular}{lllll}
\hline & $x$ & $y$ & $z$ & $U_{\text {iso }} * / U_{\text {eq }}$ \\
\hline Pt1 & $0.10189(2)$ & $0.16233(2)$ & $0.26543(2)$ & $0.00950(4)$ \\
N2 & $-0.03286(15)$ & $0.2169(2)$ & $0.12855(16)$ & $0.0120(4)$ \\
C3 & $-0.10502(19)$ & $0.1243(3)$ & $0.0838(2)$ & $0.0152(5)$ \\
H3 & -0.0934 & 0.0380 & 0.1141 & $0.018^{*}$ \\
C4 & $-0.1940(2)$ & $0.1495(3)$ & $-0.0030(2)$ & $0.0184(6)$ \\
H4 & -0.2437 & 0.0828 & -0.0320 & $0.022^{*}$
\end{tabular}




\begin{tabular}{|c|c|c|c|c|}
\hline $\mathrm{C} 5$ & $-0.2097(2)$ & $0.2756(3)$ & $-0.0476(2)$ & $0.0185(6)$ \\
\hline H5 & -0.2705 & 0.2958 & -0.1084 & $0.022 *$ \\
\hline C6 & $-0.1378(2)$ & $0.3701(3)$ & $-0.0039(2)$ & $0.0172(6)$ \\
\hline H6 & -0.1479 & 0.4561 & -0.0344 & $0.021 *$ \\
\hline $\mathrm{C} 7$ & $-0.0487(2)$ & $0.3395(2)$ & $0.0864(2)$ & $0.0131(5)$ \\
\hline N8 & $0.02411(16)$ & $0.4301(2)$ & $0.13082(18)$ & $0.0190(5)$ \\
\hline $\mathrm{H} 8 \mathrm{~A}$ & 0.0804 & 0.4087 & 0.1861 & $0.023 *$ \\
\hline H8B & 0.0154 & 0.5109 & 0.1046 & $0.023 *$ \\
\hline C9 & $0.1753(2)$ & $0.1410(3)$ & $0.1694(2)$ & $0.0165(6)$ \\
\hline H9A & 0.2434 & 0.1627 & 0.2122 & $0.020^{*}$ \\
\hline H9B & 0.1320 & 0.2033 & 0.1191 & $0.020^{*}$ \\
\hline $\mathrm{C} 10$ & $0.13895(19)$ & $0.0178(3)$ & $0.18011(19)$ & $0.0141(5)$ \\
\hline $\mathrm{H} 10$ & 0.0818 & -0.0176 & 0.1146 & $0.017 *$ \\
\hline C11 & 0.20439 (19) & -0.0853 & 0.25867 (19) & $0.0140(5)$ \\
\hline $\mathrm{H} 11 \mathrm{~A}$ & 0.1626 & -0.1585 & 0.2626 & $0.017 *$ \\
\hline H11B & 0.2511 & -0.1227 & 0.2345 & $0.017^{*}$ \\
\hline C12 & $0.26227(18)$ & $-0.0239(2)$ & $0.36696(18)$ & $0.0106(5)$ \\
\hline $\mathrm{C} 13$ & $0.22571(18)$ & 0.0934 (2) & $0.38748(19)$ & $0.0105(5)$ \\
\hline C14 & $0.27648(18)$ & 0.1489 (2) & $0.48888(19)$ & $0.0102(5)$ \\
\hline H14 & 0.2536 & 0.2298 & 0.5043 & $0.012 *$ \\
\hline $\mathrm{C} 15$ & $0.35958(18)$ & $0.0873(2)$ & $0.56683(18)$ & $0.0104(5)$ \\
\hline C16 & $0.39390(17)$ & $-0.0322(2)$ & $0.54590(18)$ & $0.0099(5)$ \\
\hline $\mathrm{C} 17$ & $0.34544(18)$ & $-0.0865(2)$ & 0.44517 (19) & $0.0105(5)$ \\
\hline H17 & 0.3691 & -0.1666 & 0.4295 & $0.013 *$ \\
\hline O18 & $0.47426(13)$ & $-0.08947(17)$ & $0.62958(13)$ & $0.0129(4)$ \\
\hline C19 & $0.52470(19)$ & $-0.1911(3)$ & $0.6034(2)$ & $0.0148(5)$ \\
\hline H19A & 0.5461 & -0.1566 & 0.5526 & $0.022 *$ \\
\hline H19B & 0.4797 & -0.2664 & 0.5719 & $0.022 *$ \\
\hline $\mathrm{H} 19 \mathrm{C}$ & 0.5831 & -0.2202 & 0.6679 & $0.022 *$ \\
\hline $\mathrm{O} 20$ & $0.40683(13)$ & $0.14275(17)$ & 0.66777 (13) & $0.0113(4)$ \\
\hline $\mathrm{C} 21$ & $0.50526(18)$ & $0.1854(2)$ & $0.6969(2)$ & $0.0124(5)$ \\
\hline $\mathrm{H} 21 \mathrm{~A}$ & 0.5375 & 0.1211 & 0.6697 & $0.015^{*}$ \\
\hline $\mathrm{H} 21 \mathrm{~B}$ & 0.5436 & 0.1867 & 0.7752 & $0.015^{*}$ \\
\hline $\mathrm{C} 22$ & $0.50842(19)$ & $0.3214(3)$ & $0.65427(19)$ & $0.0135(5)$ \\
\hline $\mathrm{O} 23$ & $0.43843(14)$ & 0.39064 (19) & $0.60292(15)$ & $0.0208(4)$ \\
\hline $\mathrm{O} 24$ & $0.60368(13)$ & $0.35149(17)$ & $0.68185(14)$ & $0.0140(4)$ \\
\hline $\mathrm{C} 25$ & $0.6206(2)$ & 0.4778 (3) & $0.6433(2)$ & $0.0177(6)$ \\
\hline $\mathrm{H} 25 \mathrm{~A}$ & 0.5806 & 0.5482 & 0.6543 & $0.021 *$ \\
\hline $\mathrm{H} 25 \mathrm{~B}$ & 0.6007 & 0.4719 & 0.5665 & $0.021 *$ \\
\hline $\mathrm{C} 26$ & $0.7291(2)$ & $0.5093(3)$ & $0.7039(2)$ & $0.0240(6)$ \\
\hline $\mathrm{H} 26 \mathrm{~A}$ & 0.7436 & 0.5898 & 0.6749 & $0.036^{*}$ \\
\hline H26B & 0.7681 & 0.4353 & 0.6978 & $0.036^{*}$ \\
\hline $\mathrm{H} 26 \mathrm{C}$ & 0.7466 & 0.5234 & 0.7788 & $0.036^{*}$ \\
\hline $\mathrm{Cl} 27$ & $0.04241(4)$ & $0.25459(6)$ & $0.37522(5)$ & 0.01518 \\
\hline
\end{tabular}


Atomic displacement parameters $\left(\AA^{2}\right)$

\begin{tabular}{|c|c|c|c|c|c|c|}
\hline & $U^{11}$ & $U^{22}$ & $U^{33}$ & $U^{12}$ & $U^{13}$ & $U^{23}$ \\
\hline Pt1 & $0.00860(6)$ & $0.00919(6)$ & $0.00852(6)$ & $0.00084(3)$ & $0.00203(4)$ & $0.00005(3)$ \\
\hline N2 & $0.0111(10)$ & $0.0127(11)$ & $0.0102(10)$ & $0.0025(9)$ & $0.0033(8)$ & $0.0001(8)$ \\
\hline $\mathrm{C} 3$ & $0.0147(13)$ & $0.0158(13)$ & 0.0155 (13) & $-0.0001(11)$ & $0.0074(11)$ & $-0.0018(11)$ \\
\hline $\mathrm{C} 4$ & $0.0147(14)$ & $0.0216(15)$ & $0.0171(13)$ & $0.0003(11)$ & $0.0058(11)$ & $-0.0074(11)$ \\
\hline $\mathrm{C} 5$ & $0.0140(13)$ & $0.0283(16)$ & $0.0101(12)$ & $0.0096(12)$ & $0.0029(10)$ & $-0.0020(11)$ \\
\hline C6 & $0.0182(14)$ & $0.0192(14)$ & 0.0134 (13) & $0.0088(11)$ & $0.0066(11)$ & $0.0030(11)$ \\
\hline $\mathrm{C} 7$ & $0.0150(13)$ & $0.0148(14)$ & $0.0122(12)$ & $0.0055(10)$ & $0.0084(11)$ & $0.0014(10)$ \\
\hline N8 & $0.0184(12)$ & $0.0116(11)$ & $0.0218(12)$ & $0.0009(9)$ & $0.0047(10)$ & $0.0043(9)$ \\
\hline C9 & $0.0136(13)$ & $0.0237(15)$ & $0.0113(12)$ & $0.0045(11)$ & $0.0051(11)$ & $0.0000(11)$ \\
\hline $\mathrm{C} 10$ & 0.0145 (13) & $0.0167(14)$ & $0.0093(11)$ & $0.0025(11)$ & $0.0039(10)$ & $-0.0050(10)$ \\
\hline $\mathrm{C} 11$ & $0.0127(13)$ & $0.0128(13)$ & $0.0129(12)$ & $0.0019(10)$ & $0.0028(10)$ & $-0.0034(10)$ \\
\hline $\mathrm{C} 12$ & $0.0103(12)$ & $0.0133(13)$ & $0.0088(11)$ & $-0.0013(10)$ & $0.0049(10)$ & $-0.0018(10)$ \\
\hline $\mathrm{C} 13$ & $0.0086(12)$ & $0.0111(13)$ & $0.0125(12)$ & $0.0000(10)$ & $0.0054(10)$ & $0.0015(10)$ \\
\hline $\mathrm{C} 14$ & $0.0106(12)$ & $0.0090(12)$ & $0.0122(12)$ & $0.0005(9)$ & $0.0063(10)$ & $-0.0004(9)$ \\
\hline $\mathrm{C} 15$ & 0.0109 (12) & $0.0128(13)$ & 0.0088 (11) & $-0.0051(10)$ & $0.0056(10)$ & $-0.0019(9)$ \\
\hline $\mathrm{C} 16$ & $0.0080(11)$ & $0.0117(13)$ & $0.0093(11)$ & $0.0004(10)$ & $0.0034(9)$ & $0.0033(9)$ \\
\hline $\mathrm{C} 17$ & 0.0123 (12) & $0.0083(12)$ & $0.0127(12)$ & $0.0015(10)$ & $0.0072(10)$ & 0.0009 (10) \\
\hline $\mathrm{O} 18$ & $0.0132(9)$ & $0.0119(9)$ & $0.0107(8)$ & $0.0044(7)$ & $0.0031(7)$ & $0.0026(7)$ \\
\hline C19 & $0.0134(13)$ & $0.0159(13)$ & $0.0154(13)$ & $0.0049(11)$ & 0.0069 (11) & $0.0043(11)$ \\
\hline $\mathrm{O} 20$ & $0.0100(9)$ & $0.0159(9)$ & $0.0079(8)$ & $-0.0030(7)$ & 0.0039 (7) & $-0.0033(7)$ \\
\hline $\mathrm{C} 21$ & 0.0103 (12) & $0.0146(13)$ & $0.0103(12)$ & $-0.0017(10)$ & $0.0031(10)$ & $-0.0010(10)$ \\
\hline $\mathrm{C} 22$ & $0.0142(13)$ & $0.0156(14)$ & $0.0088(12)$ & $-0.0022(10)$ & $0.0036(10)$ & $-0.0051(10)$ \\
\hline $\mathrm{O} 23$ & $0.0140(10)$ & $0.0204(10)$ & $0.0204(10)$ & $0.0014(8)$ & $0.0014(8)$ & $0.0044(8)$ \\
\hline $\mathrm{O} 24$ & $0.0126(9)$ & $0.0120(9)$ & $0.0178(9)$ & $-0.0010(7)$ & $0.0072(8)$ & $0.0013(7)$ \\
\hline $\mathrm{C} 25$ & $0.0212(14)$ & $0.0124(14)$ & 0.0179 (13) & $-0.0023(11)$ & $0.0076(11)$ & $0.0017(11)$ \\
\hline $\mathrm{C} 26$ & $0.0211(15)$ & $0.0194(15)$ & $0.0300(16)$ & $-0.0059(12)$ & $0.0103(13)$ & $0.0030(12)$ \\
\hline $\mathrm{C} 127$ & $0.0151(3)$ & $0.0148(3)$ & $0.0144(3)$ & $0.0038(2)$ & $0.0058(2)$ & $-0.0020(2)$ \\
\hline
\end{tabular}

Geometric parameters $\left(\AA,{ }^{\circ}\right)$

\begin{tabular}{llll}
\hline Pt1-N2 & $2.143(2)$ & $\mathrm{C} 12-\mathrm{C} 17$ & $1.389(3)$ \\
Pt1-C9 & $2.127(3)$ & $\mathrm{C} 13-\mathrm{C} 14$ & $1.399(3)$ \\
Pt1-C10 & $2.127(2)$ & $\mathrm{C} 14-\mathrm{H} 14$ & 0.9500 \\
Pt1-C13 & $2.003(2)$ & $\mathrm{C} 14-\mathrm{C} 15$ & $1.382(3)$ \\
Pt1-C127 & $2.3209(6)$ & $\mathrm{C} 15-\mathrm{C} 16$ & $1.397(4)$ \\
$\mathrm{N} 2-\mathrm{C} 3$ & $1.353(3)$ & $\mathrm{C} 15-\mathrm{O} 20$ & $1.391(3)$ \\
$\mathrm{N} 2-\mathrm{C} 7$ & $1.346(3)$ & $\mathrm{C} 16-\mathrm{C} 17$ & $1.376(3)$ \\
$\mathrm{C} 3-\mathrm{H} 3$ & 0.9500 & $\mathrm{C} 16-\mathrm{O} 18$ & 0.9500 \\
$\mathrm{C} 3-\mathrm{C} 4$ & $1.367(4)$ & $\mathrm{C} 17-\mathrm{H} 17$ & $1.425(3)$ \\
$\mathrm{C} 4-\mathrm{H} 4$ & 0.9500 & $\mathrm{O} 18-\mathrm{C} 19$ & 0.9800 \\
$\mathrm{C} 4-\mathrm{C} 5$ & $1.392(4)$ & $\mathrm{C} 19-\mathrm{H} 19 \mathrm{~A}$ & 0.9800 \\
C5-H5 & 0.9500 & $\mathrm{C} 19-\mathrm{H} 19 \mathrm{~B}$ & 0.9800 \\
$\mathrm{C} 5-\mathrm{C} 6$ & $1.363(4)$ & $\mathrm{C} 19-\mathrm{H} 19 \mathrm{C}$ & $1.416(3)$ \\
C6-H6 & 0.9500 & $\mathrm{O} 20-\mathrm{C} 21$ & 0.9900 \\
C6-C7 & $1.402(4)$ & $\mathrm{C} 21-\mathrm{H} 21 \mathrm{~A}$ &
\end{tabular}




\begin{tabular}{|c|c|c|c|}
\hline $\mathrm{C} 7-\mathrm{N} 8$ & $1.345(3)$ & $\mathrm{C} 21-\mathrm{H} 21 \mathrm{~B}$ & 0.9900 \\
\hline $\mathrm{N} 8-\mathrm{H} 8 \mathrm{~A}$ & 0.8800 & $\mathrm{C} 21-\mathrm{C} 22$ & $1.509(4)$ \\
\hline N8-H8B & 0.8800 & $\mathrm{C} 22-\mathrm{O} 23$ & $1.196(3)$ \\
\hline C9-H9A & 0.9500 & $\mathrm{C} 22-\mathrm{O} 24$ & $1.343(3)$ \\
\hline C9-H9B & 0.9500 & $\mathrm{O} 24-\mathrm{C} 25$ & $1.454(3)$ \\
\hline $\mathrm{C} 9-\mathrm{C} 10$ & $1.395(4)$ & $\mathrm{C} 25-\mathrm{H} 25 \mathrm{~A}$ & 0.9900 \\
\hline $\mathrm{C} 10-\mathrm{H} 10$ & 1.0000 & $\mathrm{C} 25-\mathrm{H} 25 \mathrm{~B}$ & 0.9900 \\
\hline $\mathrm{C} 10-\mathrm{C} 11$ & $1.514(3)$ & $\mathrm{C} 25-\mathrm{C} 26$ & $1.495(4)$ \\
\hline $\mathrm{C} 11-\mathrm{H} 11 \mathrm{~A}$ & 0.9900 & $\mathrm{C} 26-\mathrm{H} 26 \mathrm{~A}$ & 0.9800 \\
\hline $\mathrm{C} 11-\mathrm{H} 11 \mathrm{~B}$ & 0.9900 & $\mathrm{C} 26-\mathrm{H} 26 \mathrm{~B}$ & 0.9800 \\
\hline $\mathrm{C} 11-\mathrm{C} 12$ & $1.509(3)$ & $\mathrm{C} 26-\mathrm{H} 26 \mathrm{C}$ & 0.9800 \\
\hline $\mathrm{C} 12-\mathrm{C} 13$ & $1.391(4)$ & & \\
\hline $\mathrm{N} 2-\mathrm{Pt} 1-\mathrm{Cl} 27$ & $90.28(6)$ & $\mathrm{C} 13-\mathrm{C} 12-\mathrm{C} 11$ & $117.5(2)$ \\
\hline C9-Pt1-N2 & $90.24(9)$ & $\mathrm{C} 17-\mathrm{C} 12-\mathrm{C} 11$ & $121.0(2)$ \\
\hline $\mathrm{C} 9-\mathrm{Pt} 1-\mathrm{C} 10$ & $38.29(10)$ & $\mathrm{C} 17-\mathrm{C} 12-\mathrm{C} 13$ & $121.3(2)$ \\
\hline $\mathrm{C} 9-\mathrm{Pt} 1-\mathrm{Cl} 27$ & $161.33(7)$ & $\mathrm{C} 12-\mathrm{C} 13-\mathrm{Pt} 1$ & $114.69(17)$ \\
\hline $\mathrm{C} 10-\mathrm{Pt} 1-\mathrm{N} 2$ & $92.85(9)$ & $\mathrm{C} 12-\mathrm{C} 13-\mathrm{C} 14$ & $118.2(2)$ \\
\hline $\mathrm{C} 10-\mathrm{Pt} 1-\mathrm{Cl} 27$ & $160.20(7)$ & $\mathrm{C} 14-\mathrm{C} 13-\mathrm{Pt} 1$ & $127.13(19)$ \\
\hline $\mathrm{C} 13-\mathrm{Pt} 1-\mathrm{N} 2$ & $174.40(9)$ & $\mathrm{C} 13-\mathrm{C} 14-\mathrm{H} 14$ & 119.6 \\
\hline $\mathrm{C} 13-\mathrm{Pt} 1-\mathrm{C} 9$ & $87.89(10)$ & $\mathrm{C} 15-\mathrm{C} 14-\mathrm{C} 13$ & $120.7(2)$ \\
\hline $\mathrm{C} 13-\mathrm{Pt} 1-\mathrm{C} 10$ & $82.42(10)$ & $\mathrm{C} 15-\mathrm{C} 14-\mathrm{H} 14$ & 119.6 \\
\hline $\mathrm{C} 13-\mathrm{Pt} 1-\mathrm{Cl} 27$ & $93.18(7)$ & $\mathrm{C} 14-\mathrm{C} 15-\mathrm{C} 16$ & $120.5(2)$ \\
\hline $\mathrm{C} 3-\mathrm{N} 2-\mathrm{Pt} 1$ & $118.29(17)$ & $\mathrm{C} 14-\mathrm{C} 15-\mathrm{O} 20$ & $119.5(2)$ \\
\hline $\mathrm{C} 7-\mathrm{N} 2-\mathrm{Pt} 1$ & $122.69(17)$ & $\mathrm{O} 20-\mathrm{C} 15-\mathrm{C} 16$ & $120.0(2)$ \\
\hline $\mathrm{C} 7-\mathrm{N} 2-\mathrm{C} 3$ & $119.0(2)$ & $\mathrm{C} 17-\mathrm{C} 16-\mathrm{C} 15$ & $119.2(2)$ \\
\hline $\mathrm{N} 2-\mathrm{C} 3-\mathrm{H} 3$ & 118.6 & $\mathrm{O} 18-\mathrm{C} 16-\mathrm{C} 15$ & $116.5(2)$ \\
\hline $\mathrm{N} 2-\mathrm{C} 3-\mathrm{C} 4$ & $122.8(3)$ & $\mathrm{O} 18-\mathrm{C} 16-\mathrm{C} 17$ & $124.3(2)$ \\
\hline $\mathrm{C} 4-\mathrm{C} 3-\mathrm{H} 3$ & 118.6 & $\mathrm{C} 12-\mathrm{C} 17-\mathrm{H} 17$ & 120.0 \\
\hline $\mathrm{C} 3-\mathrm{C} 4-\mathrm{H} 4$ & 120.9 & $\mathrm{C} 16-\mathrm{C} 17-\mathrm{C} 12$ & $120.0(2)$ \\
\hline $\mathrm{C} 3-\mathrm{C} 4-\mathrm{C} 5$ & $118.1(3)$ & $\mathrm{C} 16-\mathrm{C} 17-\mathrm{H} 17$ & 120.0 \\
\hline $\mathrm{C} 5-\mathrm{C} 4-\mathrm{H} 4$ & 120.9 & $\mathrm{C} 16-\mathrm{O} 18-\mathrm{C} 19$ & $116.32(19)$ \\
\hline $\mathrm{C} 4-\mathrm{C} 5-\mathrm{H} 5$ & 120.1 & $\mathrm{O} 18-\mathrm{C} 19-\mathrm{H} 19 \mathrm{~A}$ & 109.5 \\
\hline $\mathrm{C} 6-\mathrm{C} 5-\mathrm{C} 4$ & $119.9(2)$ & $\mathrm{O} 18-\mathrm{C} 19-\mathrm{H} 19 \mathrm{~B}$ & 109.5 \\
\hline $\mathrm{C} 6-\mathrm{C} 5-\mathrm{H} 5$ & 120.1 & $\mathrm{O} 18-\mathrm{C} 19-\mathrm{H} 19 \mathrm{C}$ & 109.5 \\
\hline $\mathrm{C} 5-\mathrm{C} 6-\mathrm{H} 6$ & 120.2 & $\mathrm{H} 19 \mathrm{~A}-\mathrm{C} 19-\mathrm{H} 19 \mathrm{~B}$ & 109.5 \\
\hline $\mathrm{C} 5-\mathrm{C} 6-\mathrm{C} 7$ & $119.6(3)$ & $\mathrm{H} 19 \mathrm{~A}-\mathrm{C} 19-\mathrm{H} 19 \mathrm{C}$ & 109.5 \\
\hline $\mathrm{C} 7-\mathrm{C} 6-\mathrm{H} 6$ & 120.2 & $\mathrm{H} 19 \mathrm{~B}-\mathrm{C} 19-\mathrm{H} 19 \mathrm{C}$ & 109.5 \\
\hline $\mathrm{N} 2-\mathrm{C} 7-\mathrm{C} 6$ & $120.6(2)$ & $\mathrm{C} 15-\mathrm{O} 20-\mathrm{C} 21$ & $113.37(19)$ \\
\hline $\mathrm{N} 8-\mathrm{C} 7-\mathrm{N} 2$ & $118.4(2)$ & $\mathrm{O} 20-\mathrm{C} 21-\mathrm{H} 21 \mathrm{~A}$ & 109.1 \\
\hline $\mathrm{N} 8-\mathrm{C} 7-\mathrm{C} 6$ & $121.0(2)$ & $\mathrm{O} 20-\mathrm{C} 21-\mathrm{H} 21 \mathrm{~B}$ & 109.1 \\
\hline $\mathrm{C} 7-\mathrm{N} 8-\mathrm{H} 8 \mathrm{~A}$ & 120.0 & $\mathrm{O} 20-\mathrm{C} 21-\mathrm{C} 22$ & $112.4(2)$ \\
\hline $\mathrm{C} 7-\mathrm{N} 8-\mathrm{H} 8 \mathrm{~B}$ & 120.0 & $\mathrm{H} 21 \mathrm{~A}-\mathrm{C} 21-\mathrm{H} 21 \mathrm{~B}$ & 107.9 \\
\hline $\mathrm{H} 8 \mathrm{~A}-\mathrm{N} 8-\mathrm{H} 8 \mathrm{~B}$ & 120.0 & $\mathrm{C} 22-\mathrm{C} 21-\mathrm{H} 21 \mathrm{~A}$ & 109.1 \\
\hline Pt1-C9-H9A & 107.3 & $\mathrm{C} 22-\mathrm{C} 21-\mathrm{H} 21 \mathrm{~B}$ & 109.1 \\
\hline $\mathrm{Pt} 1-\mathrm{C} 9-\mathrm{H} 9 \mathrm{~B}$ & 91.7 & $\mathrm{O} 23-\mathrm{C} 22-\mathrm{C} 21$ & $126.4(2)$ \\
\hline $\mathrm{H} 9 \mathrm{~A}-\mathrm{C} 9-\mathrm{H} 9 \mathrm{~B}$ & 120.0 & $\mathrm{O} 23-\mathrm{C} 22-\mathrm{O} 24$ & $125.2(2)$ \\
\hline $\mathrm{C} 10-\mathrm{C} 9-\mathrm{Pt} 1$ & $70.86(15)$ & $\mathrm{O} 24-\mathrm{C} 22-\mathrm{C} 21$ & $108.4(2)$ \\
\hline
\end{tabular}




\begin{tabular}{|c|c|}
\hline $\mathrm{C} 10-\mathrm{C} 9-\mathrm{H} 9 \mathrm{~A}$ & 120.0 \\
\hline $\mathrm{C} 10-\mathrm{C} 9-\mathrm{H} 9 \mathrm{~B}$ & 120.0 \\
\hline $\mathrm{Pt} 1-\mathrm{C} 10-\mathrm{H} 10$ & 115.6 \\
\hline $\mathrm{C} 9-\mathrm{C} 10-\mathrm{Pt} 1$ & $70.85(15)$ \\
\hline $\mathrm{C} 9-\mathrm{C} 10-\mathrm{H} 10$ & 115.6 \\
\hline $\mathrm{C} 9-\mathrm{C} 10-\mathrm{C} 11$ & $122.4(2)$ \\
\hline $\mathrm{C} 11-\mathrm{C} 10-\mathrm{Pt} 1$ & $107.81(16)$ \\
\hline $\mathrm{C} 11-\mathrm{C} 10-\mathrm{H} 10$ & 115.6 \\
\hline $\mathrm{C} 10-\mathrm{C} 11-\mathrm{H} 11 \mathrm{~A}$ & 109.6 \\
\hline $\mathrm{C} 10-\mathrm{C} 11-\mathrm{H} 11 \mathrm{~B}$ & 109.6 \\
\hline $\mathrm{H} 11 \mathrm{~A}-\mathrm{C} 11-\mathrm{H} 11 \mathrm{~B}$ & 108.1 \\
\hline $\mathrm{C} 12-\mathrm{C} 11-\mathrm{C} 10$ & $110.2(2)$ \\
\hline $\mathrm{C} 12-\mathrm{C} 11-\mathrm{H} 11 \mathrm{~A}$ & 109.6 \\
\hline $\mathrm{C} 12-\mathrm{C} 11-\mathrm{H} 11 \mathrm{~B}$ & 109.6 \\
\hline $\mathrm{Pt} 1-\mathrm{N} 2-\mathrm{C} 3-\mathrm{C} 4$ & $179.4(2)$ \\
\hline $\mathrm{Pt} 1-\mathrm{N} 2-\mathrm{C} 7-\mathrm{C} 6$ & $179.41(18)$ \\
\hline $\mathrm{Pt} 1-\mathrm{N} 2-\mathrm{C} 7-\mathrm{N} 8$ & $1.5(3)$ \\
\hline $\mathrm{Pt} 1-\mathrm{C} 9-\mathrm{C} 10-\mathrm{C} 11$ & $-99.4(2)$ \\
\hline $\mathrm{Pt} 1-\mathrm{C} 10-\mathrm{C} 11-\mathrm{C} 12$ & $-28.5(2)$ \\
\hline $\mathrm{Pt} 1-\mathrm{C} 13-\mathrm{C} 14-\mathrm{C} 15$ & $177.68(18)$ \\
\hline $\mathrm{N} 2-\mathrm{C} 3-\mathrm{C} 4-\mathrm{C} 5$ & $1.0(4)$ \\
\hline $\mathrm{C} 3-\mathrm{N} 2-\mathrm{C} 7-\mathrm{C} 6$ & $-1.3(4)$ \\
\hline $\mathrm{C} 3-\mathrm{N} 2-\mathrm{C} 7-\mathrm{N} 8$ & $-179.2(2)$ \\
\hline $\mathrm{C} 3-\mathrm{C} 4-\mathrm{C} 5-\mathrm{C} 6$ & $-0.7(4)$ \\
\hline $\mathrm{C} 4-\mathrm{C} 5-\mathrm{C} 6-\mathrm{C} 7$ & $-0.5(4)$ \\
\hline $\mathrm{C} 5-\mathrm{C} 6-\mathrm{C} 7-\mathrm{N} 2$ & $1.5(4)$ \\
\hline $\mathrm{C} 5-\mathrm{C} 6-\mathrm{C} 7-\mathrm{N} 8$ & $179.4(3)$ \\
\hline $\mathrm{C} 7-\mathrm{N} 2-\mathrm{C} 3-\mathrm{C} 4$ & $0.0(4)$ \\
\hline $\mathrm{C} 9-\mathrm{C} 10-\mathrm{C} 11-\mathrm{C} 12$ & $49.7(3)$ \\
\hline $\mathrm{C} 10-\mathrm{C} 11-\mathrm{C} 12-\mathrm{C} 13$ & $21.0(3)$ \\
\hline $\mathrm{C} 10-\mathrm{C} 11-\mathrm{C} 12-\mathrm{C} 17$ & $-162.9(2)$ \\
\hline $\mathrm{C} 11-\mathrm{C} 12-\mathrm{C} 13-\mathrm{Pt} 1$ & $-1.4(3)$ \\
\hline $\mathrm{C} 11-\mathrm{C} 12-\mathrm{C} 13-\mathrm{C} 14$ & $177.6(2)$ \\
\hline $\mathrm{C} 11-\mathrm{C} 12-\mathrm{C} 17-\mathrm{C} 16$ & $-176.2(2)$ \\
\hline $\mathrm{C} 12-\mathrm{C} 13-\mathrm{C} 14-\mathrm{C} 15$ & $-1.2(4)$ \\
\hline
\end{tabular}

$\mathrm{C} 22-\mathrm{O} 24-\mathrm{C} 25 \quad 116.0(2)$

$\mathrm{O} 24-\mathrm{C} 25-\mathrm{H} 25 \mathrm{~A}$

$\mathrm{O} 24-\mathrm{C} 25-\mathrm{H} 25 \mathrm{~B}$

$\mathrm{O} 24-\mathrm{C} 25-\mathrm{C} 26$

$\mathrm{H} 25 \mathrm{~A}-\mathrm{C} 25-\mathrm{H} 25 \mathrm{~B}$

C26-C $25-\mathrm{H} 25 \mathrm{~A}$

$\mathrm{C} 26-\mathrm{C} 25-\mathrm{H} 25 \mathrm{~B}$

$\mathrm{C} 25-\mathrm{C} 26-\mathrm{H} 26 \mathrm{~A}$

$\mathrm{C} 25-\mathrm{C} 26-\mathrm{H} 26 \mathrm{~B}$

$\mathrm{C} 25-\mathrm{C} 26-\mathrm{H} 26 \mathrm{C}$

$\mathrm{H} 26 \mathrm{~A}-\mathrm{C} 26-\mathrm{H} 26 \mathrm{~B}$

$\mathrm{H} 26 \mathrm{~A}-\mathrm{C} 26-\mathrm{H} 26 \mathrm{C}$

$\mathrm{H} 26 \mathrm{~B}-\mathrm{C} 26-\mathrm{H} 26 \mathrm{C}$

110.1

110.1

$107.8(2)$

108.5

110.1

110.1

109.5

109.5

109.5

109.5

109.5

109.5

$\mathrm{C} 13-\mathrm{C} 12-\mathrm{C} 17-\mathrm{C} 16$

$-0.2(4)$

$-0.5(4)$

$\mathrm{C} 13-\mathrm{C} 14-\mathrm{C} 15-\mathrm{C} 16$

$\mathrm{C} 13-\mathrm{C} 14-\mathrm{C} 15-\mathrm{O} 20$

$-178.1(2)$

$\mathrm{C} 14-\mathrm{C} 15-\mathrm{C} 16-\mathrm{C} 17$

$\mathrm{C} 14-\mathrm{C} 15-\mathrm{C} 16-\mathrm{O} 18$

1.9 (4)

$-177.0(2)$

$-115.9(2)$

$-1.5(4)$

$-164.7(2)$

$83.1(3)$

66.4 (3)

$-177.47(19)$

$1.5(4)$

$16.5(3)$

$177.3(2)$

$179.5(2)$

$0.6(3)$

$1.2(4)$

$-177.57(19)$

$177.5(2)$

$165.5(2)$

$-1.3(4)$

Hydrogen-bond geometry $\left(A,{ }^{o}\right)$

$\mathrm{Cg} 2$ is the centroid of the $\mathrm{C} 12-\mathrm{C} 17$ ring.

\begin{tabular}{lllll}
\hline$D-\mathrm{H} \cdots A$ & $D-\mathrm{H}$ & $\mathrm{H} \cdots A$ & $D \cdots A$ & $D-\mathrm{H} \cdots A$ \\
\hline $\mathrm{C} 17-\mathrm{H} 17 \cdots \mathrm{O} 24^{\mathrm{i}}$ & 0.95 & 2.60 & $3.501(3)$ & 159 \\
$\mathrm{C} 25-\mathrm{H} 25 B \cdots \mathrm{O} 23^{\mathrm{ii}}$ & 0.99 & 2.60 & $3.449(3)$ & 144 \\
$\mathrm{~N} 8-\mathrm{H} 8 B \cdots \mathrm{Cl} 27^{\mathrm{iii}}$ & 0.88 & 2.67 & $3.413(2)$ & 143 \\
$\mathrm{C} 19-\mathrm{H} 19 A \cdots C g 2^{\mathrm{i}}$ & 0.98 & 2.63 & $3.476(3)$ & 145 \\
\hline
\end{tabular}

Symmetry codes: (i) $-x+1,-y,-z+1$; (ii) $-x+1,-y+1,-z+1$; (iii) $-x, y+1 / 2,-z+1 / 2$. 